\title{
Statin use and prognosis of lung cancer: a systematic review and meta-analysis of observational studies and randomized controlled trials
}

This article was published in the following Dove Medical Press journal: Drug Design, Development and Therapy

\author{
Dao-Kui Xia',* \\ Zhi-Gang $\mathrm{Hu}^{2,3, *}$ \\ Yu-Feng Tian ${ }^{4, *}$ \\ Fan-Jun Zeng ${ }^{2,3}$ \\ 'Department of Thoracic Surgery, \\ Yichang Central People's Hospital, \\ Yichang, PR China; ${ }^{2}$ Department of \\ Respiratory Medicine, Respiratory \\ Disease Research Institute of \\ China, Three Gorges University, \\ Yichang, PR China; ${ }^{3}$ Department \\ of Respiratory Medicine, Yichang \\ Central People's Hospital, Yichang, \\ PR China; ${ }^{4}$ Department of Academic \\ Management, Clinical Research \\ Center, Three Gorges University, \\ Yichang, PR China \\ *These authors contributed equally \\ to this work
}

Background: Previous clinical studies reported inconsistent results on the associations of statins with the mortality and survival of lung cancer patients. This review and meta-analysis summarized the impact of statins on mortality and survival of lung cancer patients.

Materials and methods: Eligible papers of this meta-analysis were searched by using PubMed, EMBASE, and Cochrane until July 2017. Primary end points were the mortality (all-cause mortality and cancer-specific mortality) and survival (progression-free survival and overall survival) of patients with statin use. Secondary end points were overall response rate and safety. The random-effects model was used to calculate pooled HRs and 95\% CIs.

Results: Seventeen studies involving 98,445 patients were included in the meta-analysis. In observational studies, the pooled HR indicated that statins potentially decreased the cancerspecific mortality and promoted the overall survival of lung cancer patients. Statins showed an association with decreased all-cause mortality in cohort studies ( $\mathrm{HR}=0.77,95 \% \mathrm{CI}$ : 0.59-0.99), but not in case-control studies ( $\mathrm{HR}=0.75,95 \% \mathrm{CI}$ : 0.50-1.10). However, statin use showed no impact on mortality and overall survival in randomized controlled trials. Meanwhile, this meta-analysis indicated that statin use did not affect the progression-free survival of lung cancer patients in observational studies and randomized controlled trials. In addition, statins potentially enhanced the effects of tyrosine kinase inhibitors ( $\mathrm{HR}=0.86,95 \% \mathrm{CI}$ : $0.76-0.98$ ) and chemotherapy ( $\mathrm{HR}=0.86,95 \% \mathrm{CI}: 0.81-0.91)$ on the overall survival of patients with nonsmall-cell lung cancer, but did not increase overall response rate and toxicity.

Conclusion: Statins were potentially associated with the decreasing risk of mortality and the improvement of overall survival in observational studies but not in randomized controlled trials.

Keywords: statin, lung cancer, mortality, survival, statins, lung cancer, prognosis

\section{Introduction}

In PR China, 4,291,600 incidents of and 2,814,200 deaths from cancer were estimated in 2015. Lung cancer was the leading cancer of morbidity $(733,300)$ and mortality $(610,200)$. Compared with urban patients, rural patients with lung cancer with high smoking prevalence and low income have a higher incident rate and a poorer 5-year survival rate. ${ }^{1}$ Although platinum-based chemotherapy and tyrosine kinase inhibitors (TKIs) improve the overall survival of patients with non-small-cell lung cancer (NSCLC), these therapeutic options bring huge financial and mental burden to these patients. Therefore, developing simple and cheap agents is necessary.

As a class of frequently prescribed drugs worldwide, statins are widely used to reduce cholesterolemia and manage cardiocerebrovascular diseases because of their cost-effectiveness and safety. ${ }^{2,3}$ Long-term statin use has effectively decreased the
Department of Respiratory Medicine, Respiratory Disease Research Institute of China, Three Gorges University, No 183 Yiling Road, Yichang 443003, PR China Email hxq910813@163.com 
disease-specific mortality and all-cause mortality of cardiocerebrovascular diseases. Recently, the potential anticancer effects of statins have attracted increasing attention. In in vitro lung cancer cells, statins induced apoptosis, ${ }^{4}$ reduced metastasis, ${ }^{5}$ and inhibited angiogenesis and tumor growth. ${ }^{6,7}$ Furthermore, statins overcame platinum and TKIs resistance in lung cancer cells and in patients..$^{8-10}$ These results supported that statins can be applied to treat lung cancer alone and in combination with chemotherapy and TKIs.

In the past decade, many studies and meta-analyses investigated the relationship of statins to the mortality and survival of several types of patients with cancer, including lung cancer, ${ }^{11-13}$ breast cancer, ${ }^{14}$ skin cancer, ${ }^{15}$ renal cell carcinoma, ${ }^{16}$ and colorectal cancer. ${ }^{17}$ However, their results were controversial. Meta-analyses demonstrated that statin use is associated with the reduced mortality and prolonged survival of patients with breast cancer, ${ }^{14}$ renal cell carcinoma ${ }^{16}$ and colorectal cancer, ${ }^{17}$ but had no impact on skin cancer. ${ }^{15}$ However, no meta-analysis was performed to assess the therapeutic effect of statins on the mortality and survival of patients with lung cancer. Existing studies on the relationship between statins and lung cancer harbored inconsistent results. ${ }^{11-13}$ As the currently largest cohort study, a Danish study revealed that prediagnosis statin use $(\mathrm{HR}=0.87$, 95\% CI: 0.83-0.92) reduced cancer-specific mortality. ${ }^{11}$ Another large-scale cohort study, which also reported protective effects of postdiagnosis statin use $(\mathrm{HR}=0.88,95 \%$ CI: 0.83-0.93), observed similar results in patients who received prediagnosis statins. ${ }^{12}$ However, a large randomized controlled trial (RCT) demonstrated that statin use did not significantly improve the progression-free survival and overall survival of patients with lung cancer. ${ }^{13}$ Thus, this meta-analysis summarized all eligible papers to evaluate the relationship among statins, lung cancer mortality, and survival. Subgroup analyses were performed to clarify the relationship further, and the overall response rate and safety of statin use were assessed.

\section{Materials and methods}

\section{Data sources and search strategies}

Eligible papers until July 2017 were searched by using PubMed, EMBASE, and Cochrane. We performed this search using the following keywords: "statin or statins or hydroxymethylglutaryl coenzyme reductase inhibitor or HMG CoA reductase inhibitors", "lung cancer or lung carcinoma", and "mortality or survival". The search was limited to English language articles. All reference lists of all articles and available reviews were screened manually to identify further other potential studies. Two independent investigators (Zhi-Gang $\mathrm{Hu}$ and Dao-Kui Xia) reviewed all available studies, and any differences were resolved with counsel. When two or more articles had probable overlap, we included the article with the larger number of patients.

\section{Inclusion criteria}

The following inclusion criteria were used to evaluate whether the study was included in this meta-analysis: 1) the study evaluated the effect of statin use in prediagnosis or postdiagnosis of lung cancer; 2) the study reported mortality or survival from lung cancer; 3 ) the study reported the hazard risk (HR) estimates of mortality and survival or had information to calculate them.

\section{Data extraction}

Two independent investigators (Zhi-Gang Hu and Dao-Kui Xia) extracted the data of all relevant studies. The extracted data included the following information: author, year of publication, country, follow-up time, study design, no of patients, cancer type, timing of statin use, treatment for lung cancer, outcome, confounding factors for matching or adjustments. If a relevant study reported many HRs of the relationship for the same result, the adjusted HR or multivariate HR was used. In situations when only crude HR was given, the unadjusted HR was retrieved. If one study reported the HR of different study designs, the HR of each study design was extracted. Primary end points were mortality (all-cause mortality and cancer-specific mortality) and survival (progression-free survival and overall survival) of lung cancer patients with statin use. Secondary end points were overall response rate and safety.

\section{Patient and public involvement}

Because all data were derived from published documents, no patients were involved in the development of the research question, outcome measures, and study design. Also, there were no patients involved in the recruitment to and conduct of the study. The authors had no plans to disseminate the results to participants of this study.

\section{Study quality assessment}

For cohort and case-control studies, the authors evaluated the methodological quality of all relevant studies using the Newcastle-Ottawa scale. ${ }^{18}$ Every paper was assessed based on three items, which included selection, comparability, and exposure (or outcome). The authors considered the included studies with seven stars or more as high quality. 
For RCTs, the Cochrane risk of bias tool was used to evaluate methodological quality. ${ }^{19}$ Zhi-Gang $\mathrm{Hu}$ and Wen-Xin Li, respectively, performed study quality assessment. Any discrepancy was solved with consensus involving a third investigator (Fan-Jun Zeng).

\section{Statistical methods}

The pooled HR and 95\% CIs were used to evaluate the value of statin use in the mortality and survival of lung cancer. Overall response rate was assessed by pooled OR estimates and corresponding 95\% CI. In our study, some analyses possibly harbor significant heterogeneity. Thus, the pooled estimates were obtained from the random-effects model where the restricted maximum likelihood estimator was available to assess inter-study heterogeneity. ${ }^{20,21}$ The Cochrane $Q$ and $I^{2}$ statistics were applied to evaluate between-study heterogeneity. $P<0.10$ for $Q$ test and $I^{2}>50 \%$ indicated significant heterogeneity. The source of significant heterogeneity was analyzed through subgroup analysis. Study design influenced the credibility of the results. Therefore, we performed a subgroup analysis based on study design to evaluate the impact of statin use on lung cancer, as the two major types of lung cancer, small cell lung cancer (SCLC) and NSCLC, possess different gene mutations and treatment options, with different overall survival rate. This meta-analysis was aimed to assess the efficacy of statin use for lung cancer based on different cancer type and combined treatment options. Some studies evaluated whether timing of statin use influences its effect on the mortality of lung cancer patients. Subgroup analysis based on timing of statin use was available to guide the selection of timing of statin use in treating lung cancer. Survival benefit of statin use in treating lung cancer was supposed to be attributed to reduced mortality due to the mortality of comorbidity of these patients. This study also evaluated whether comorbidity affects the effect of statin use on the mortality and survival of lung cancer patients, including chronic obstructive pulmonary disease, coronary artery disease, and stroke. Funnel plot asymmetry reflected potential published bias. The potential published bias was evaluated through Begg's and Egger's regression tests. When the authors found publication bias, the potential influence of this bias was assessed by using the trim-and-fill method. All statistical analyses were carried out by using Stata version 10.0 .

\section{Results}

\section{Identification of eligible studies}

The flow diagram for study selection is showed in Figure 1. The initial search yielded 881 records from
PubMed, EMBASE, Web of Science, and the Cochrane library. After removing duplicate studies, we reviewed the title/abstract of 878 studies and excluded 851 studies. Subsequently, 27 potential studies were selected for the full-text review with 10 studies excluded. Finally, a total of 17 studies $^{11-13,22-35}$ were included in the meta-analysis, with 14 observational studies ${ }^{11,12,22-26,28-31,33-35}$ and 3 RCTs. ${ }^{13,27,32}$

\section{Characteristics of the studies}

Seventeen papers involving 98,445 patients were included in the meta-analysis. ${ }^{11-13,22-35}$ The detailed characteristics and clinical outcomes of relevant studies are summarized in Tables 1 and 2. There were eleven cohort studies, ${ }^{11,12,22-24,28-30,33-35}$ three case-control studies, ${ }^{25,26,31}$ and three RCTs. ${ }^{13,27,32}$ Four studies simultaneously reported the outcomes of cohort study and case-control studies. ${ }^{11,12,22,23}$ Two studies evaluated the relationship between prediagnosis statins and mortality of patients with lung cancer. ${ }^{11,25}$ One paper contained data on both prediagnosis and postdiagnosis statins. ${ }^{12}$ Other 14 studies reported outcomes of postdiagnosis statin use. ${ }^{13,22-24,26-35}$ Of the 17 included studies, 8 studies were conducted in America, ${ }^{24,26,28,29,31,33-35} 5$ in Asia, ${ }^{22,23,25,27,32}$ and the remaining 4 in Europe. ${ }^{11-13,30}$ Twelve studies clearly reported on cancer types, including 10 studies of NSCLC ${ }^{22,23,27,28,30-35}$ and 2 studies of SCLC. ${ }^{13,24}$ The methodological quality of the observational studies $(n=14)$ and RCTs $(n=3)$ was assessed using the Newcastle-Ottawa scale and the Cochrane risk of bias tool, respectively (Table S1 and Figure S1).

\section{Mortality}

\section{All-cause mortality}

Seven HR estimates of lung cancer all-cause mortality were found, involving 44,247 patients (Figure 2A). ${ }^{12,22,25,27,33}$ The pooled $(\mathrm{HR}=0.77,95 \% \mathrm{CI}: 0.65-0.93)$ suggested that statins were related to the reduction of all-cause mortality in lung cancer (Table 3 ). In the subgroup analysis by study design, a significant reduction in all-cause mortality was found in cohort studies (HR $=0.77$, 95\% CI: 0.59-0.99), but not in case-control studies $(\mathrm{HR}=0.75,95 \% \mathrm{CI}: 0.50-1.10)$ and RCTs $(\mathrm{HR}=1.03,95 \% \mathrm{CI}: 0.58-1.81)$. Both prediagnosis $(\mathrm{HR}=0.90,95 \% \mathrm{CI}: 0.97-0.93)$ and postdiagnosis $(\mathrm{HR}=0.71$, 95\% CI: 0.59-0.85) statin use were associated with the reduction of all-cause mortality. We also performed some other subgroup analyses, including NSCLC (HR $=0.62,95 \% \mathrm{CI}$ : 0.56-0.69), combined TKIs therapy ( $\mathrm{HR}=0.60,95 \% \mathrm{CI}$ : $0.55-0.66)$, and combined chemotherapy (HR $=0.63,95 \%$ CI: $0.57-0.71)$. In addition, we assessed whether comorbidity affected the effect of statin use for all-cause mortality. 


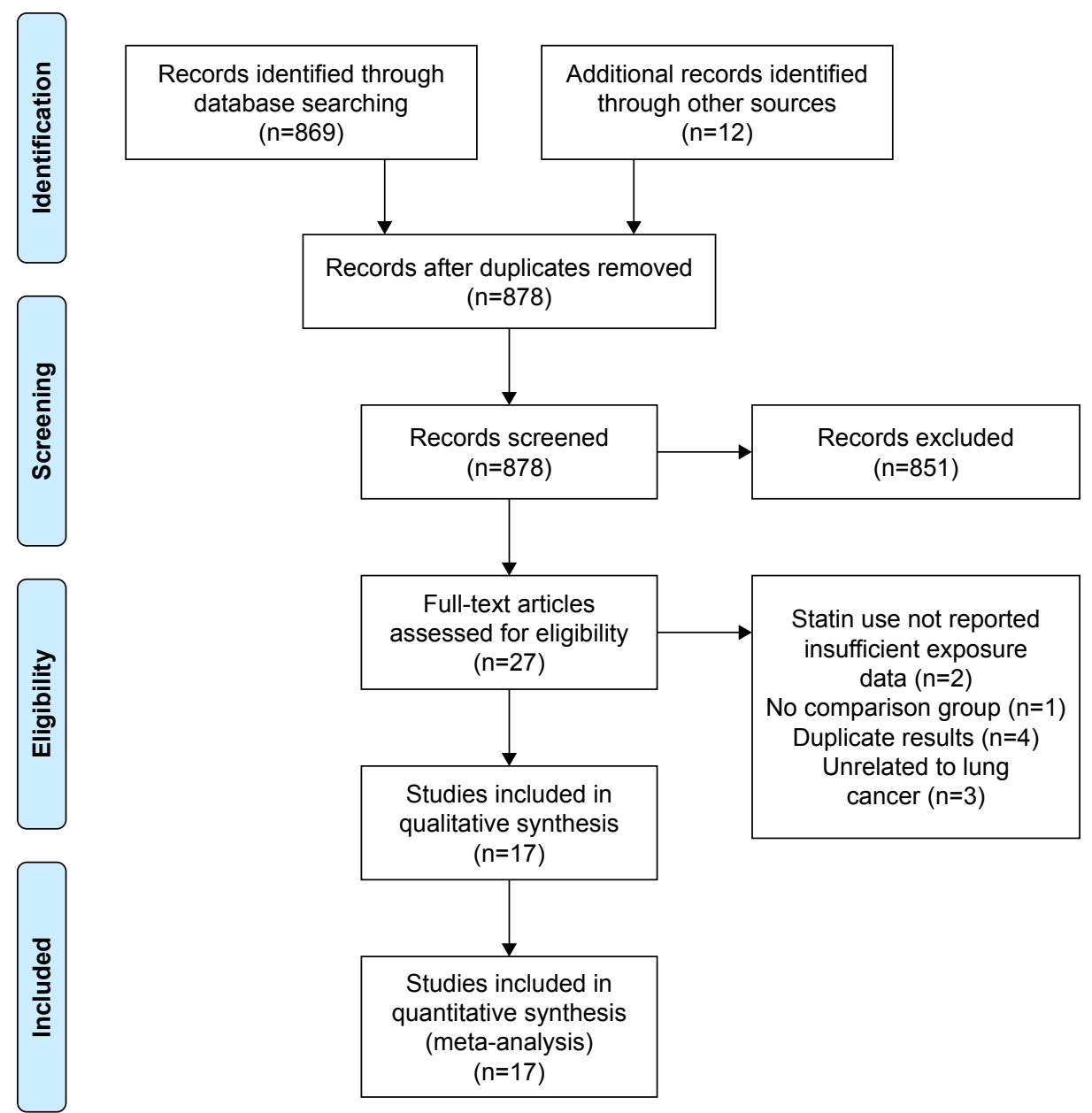

Figure I Flow diagram of the selection of publications included in the meta-analysis.

These comorbidities comprised chronic obstructive pulmonary disease, coronary artery disease, and stroke. Similar HR estimates were found in lung cancer patients with comorbidities and without comorbidities (Figure S2).

There was a high heterogeneity in all-cause mortality $\left(I^{2}=96.8 \%, P<0.001\right)$. By heterogeneity test and subgroup analysis, one study was deemed to be the main heterogeneity source..$^{22}$ When we removed this study, heterogeneity $\left(I^{2}=0 \%, P=0.53\right)$ decreased with pooled $(\mathrm{HR}=0.90,95 \% \mathrm{CI}$ : $0.87-0.93)$. In the subgroup analyses without this study, all the above results were not substantially changed with low heterogeneity (data not shown).

\section{Cancer-specific mortality}

Six HR estimates of cancer-specific mortality for patients with lung cancer were found, involving 74,034 patients (Figure 2B). ${ }^{11,12,29}$ Significant relationship was found between statins and cancer-specific mortality (HR $=0.89,95 \% \mathrm{CI}$ : $0.84-0.94)$ with high heterogeneity $\left(I^{2}=63.6 \%, P<0.001\right.$; Table 4). When stratified by study design, we observed a significant reduction of cancer-specific mortality in cohort studies (HR $=0.91,95 \%$ CI: 0.84-0.99) and case-control studies ( $\mathrm{HR}=0.85,95 \% \mathrm{CI}: 0.78-0.93$ ). In addition, the risk of cancer-specific mortality significantly decreased in prediagnosis ( $\mathrm{HR}=0.71,95 \% \mathrm{CI}: 0.59-0.85$ ) and postdiagnosis (HR $=0.90,95 \%$ CI: 0.87-0.93) statin use.

One study was considered as the main heterogeneity source through heterogeneity test and subgroup analysis, which reported the only negative result of cancer-specific mortality. ${ }^{29}$ When we dropped this study, a decreased risk of cancer-specific mortality (HR $=0.86,95 \%$ CI: $0.84-0.89$ ) was observed with low heterogeneity $\left(I^{2}=0 \%, P=0.49\right)$. In the subgroup analyses without this study, all the above results were not substantially changed with low heterogeneity (data not shown).

\section{Survival}

\section{Progression-free survival}

Six HR estimates of progression-free survival were found, involving 2,389 patients (Figure 2C). ${ }^{13,27,30,32,34,35}$ There was 


\begin{tabular}{|c|c|c|c|c|c|c|c|}
\hline & 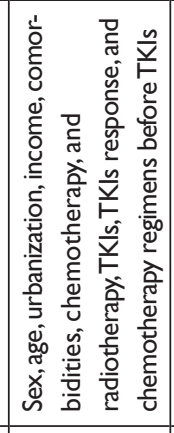 & $\frac{1}{z}$ & 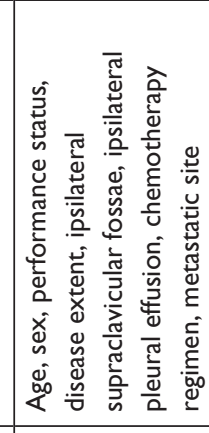 & 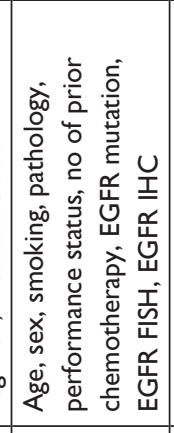 & 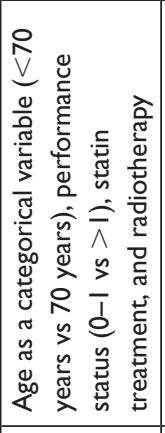 & 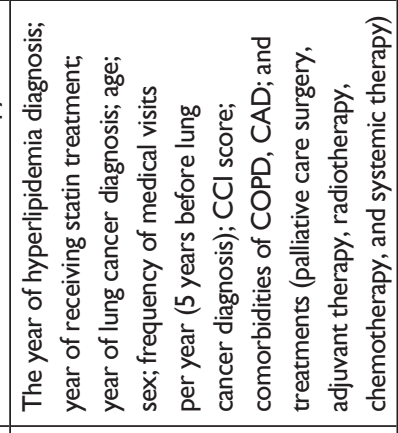 & 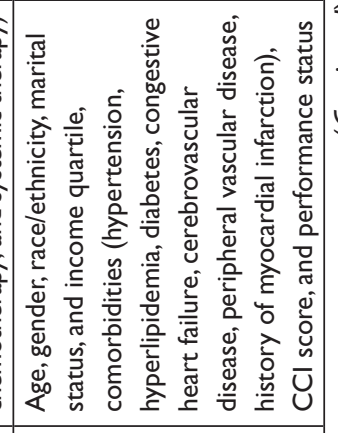 \\
\hline & 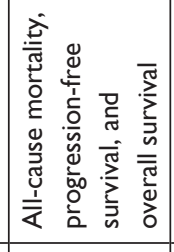 & 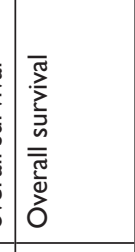 & 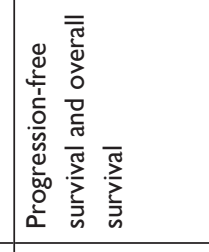 & 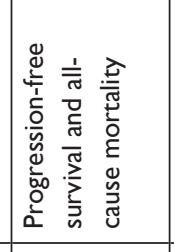 & 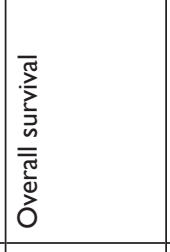 & 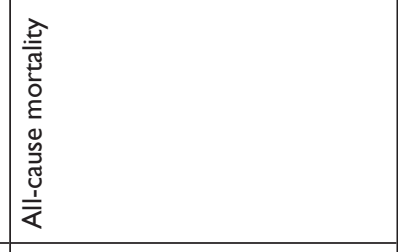 & 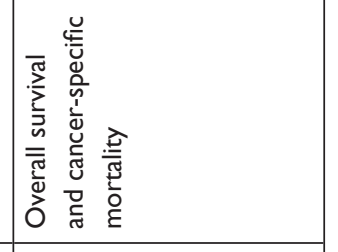 \\
\hline 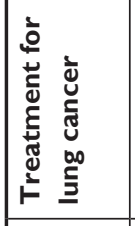 & 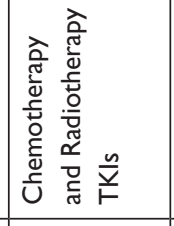 & 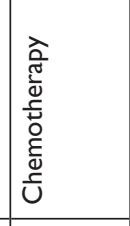 & 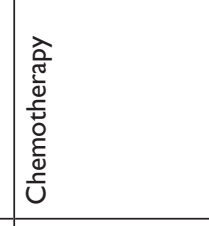 & $\frac{n}{\ddot{F}}$ & 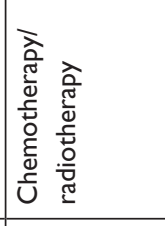 & 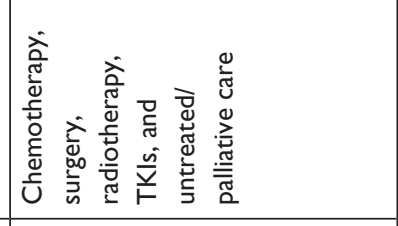 & 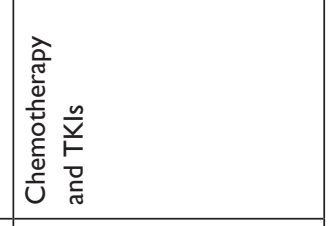 \\
\hline 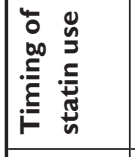 & 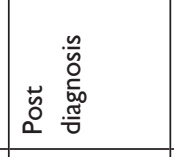 & 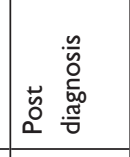 & 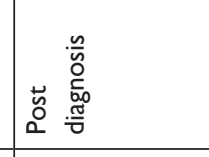 & 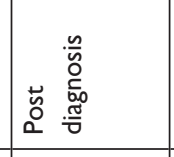 & 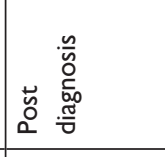 & 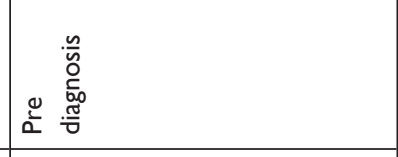 & 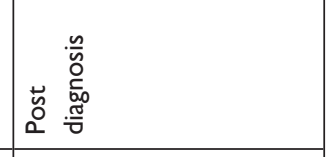 \\
\hline 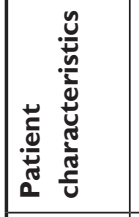 & \begin{tabular}{|l}
$u$ \\
$u$ \\
ż
\end{tabular} & \begin{tabular}{|l}
$u$ \\
$\mathbf{u}$ \\
$\underline{z}$ \\
\end{tabular} & 岮 & $\begin{array}{l}u \\
u \\
u \\
z\end{array}$ & U⿱ 己丸 & 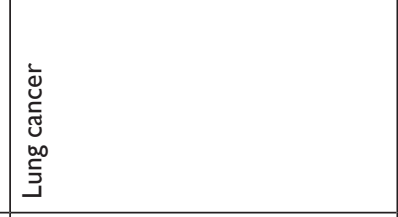 & 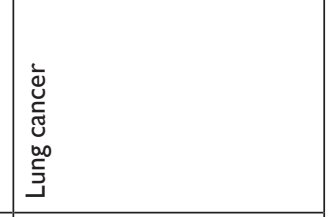 \\
\hline 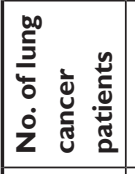 & 柋 & 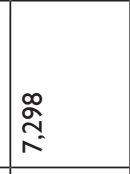 & $\begin{array}{l}\text { W } \\
\infty\end{array}$ & œ & 总 & 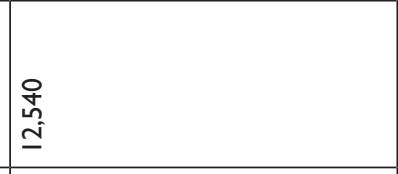 & $\frac{\infty}{\omega}$ \\
\hline 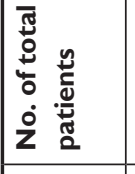 & 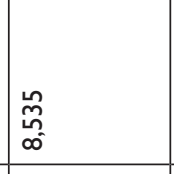 & ָָ & $\begin{array}{l}0 \\
\vdots \\
\infty\end{array}$ & $\infty$ & 芯 & 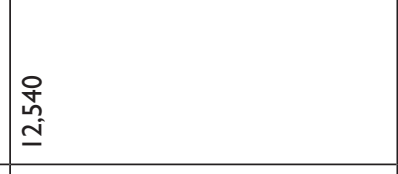 & $\frac{\infty}{\omega n}$ \\
\hline 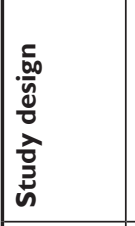 & 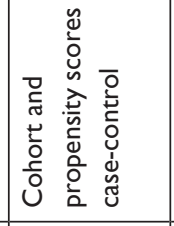 & 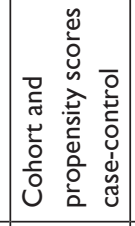 & tu & $\begin{array}{l}\underline{y} \\
\propto \\
\end{array}$ & $\begin{array}{l} \\
\frac{0}{0} \\
\\
\end{array}$ & 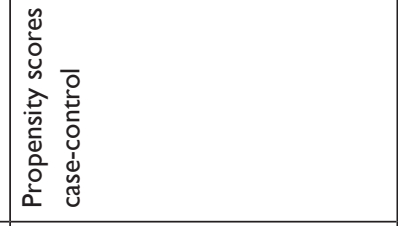 & 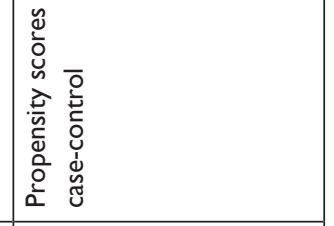 \\
\hline 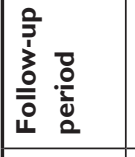 & 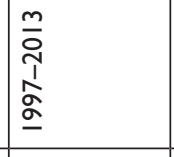 & 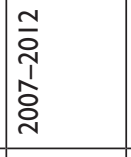 & 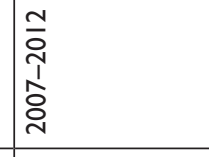 & 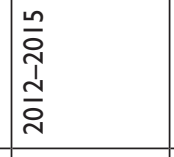 & 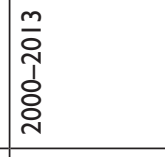 & 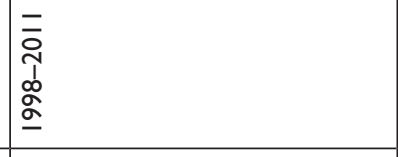 & 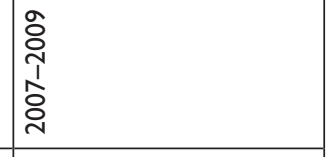 \\
\hline ט & 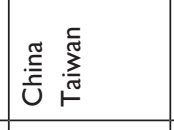 & 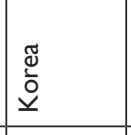 & כ & 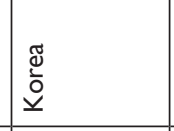 & 芯 & 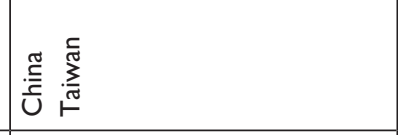 & 芯 \\
\hline 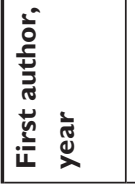 & 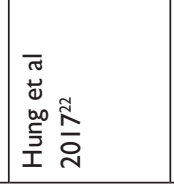 & 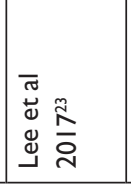 & 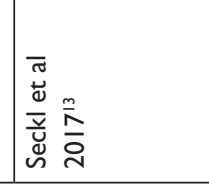 & 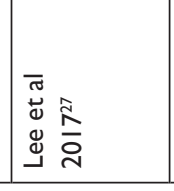 & 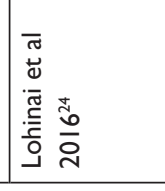 & 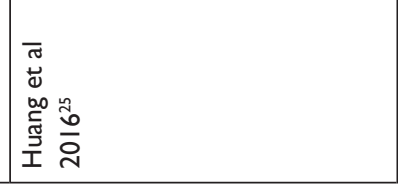 & 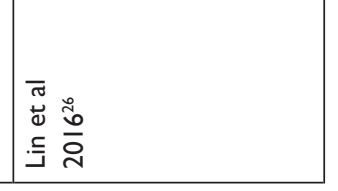 \\
\hline
\end{tabular}




\begin{tabular}{|c|c|c|c|c|c|c|c|}
\hline 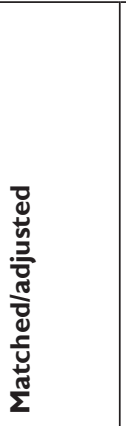 & 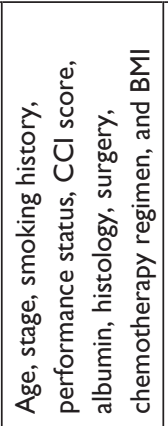 & 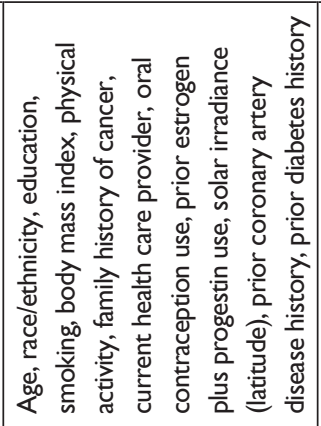 & 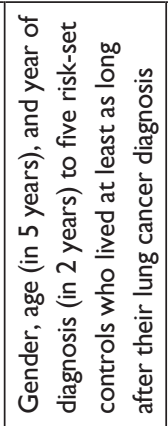 & 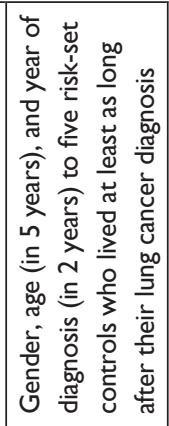 & 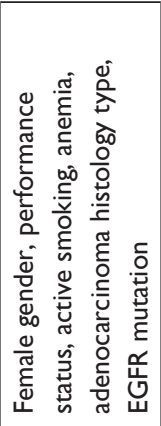 & 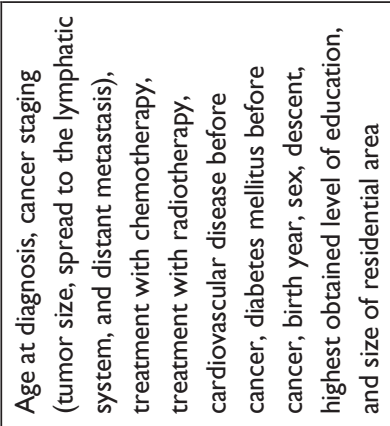 & \begin{tabular}{|l} 
\\
$\overleftarrow{z}$
\end{tabular} \\
\hline 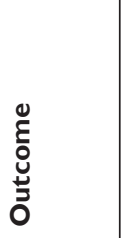 & 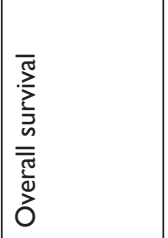 & 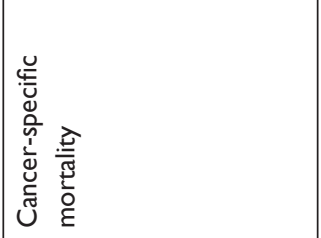 & 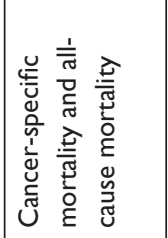 & 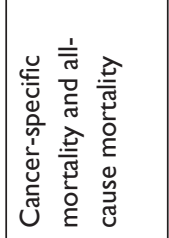 & 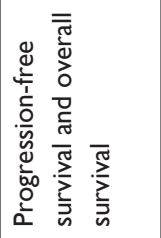 & 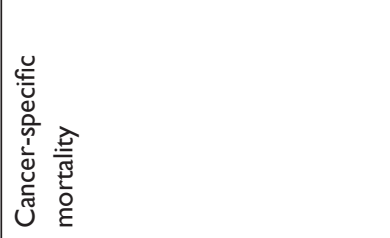 & 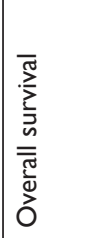 \\
\hline 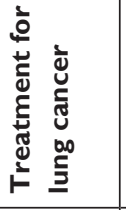 & 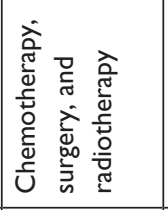 & $\S$ & 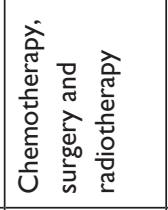 & 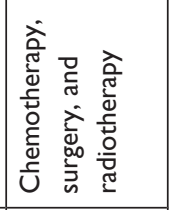 & $\frac{n}{F}$ & $\S$ & 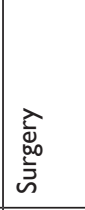 \\
\hline 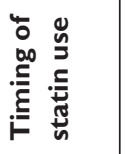 & 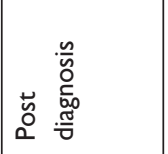 & 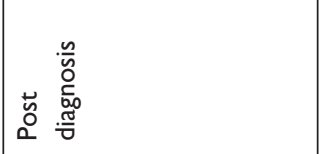 & 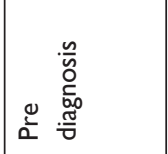 & 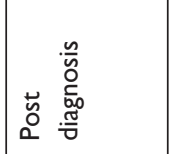 & 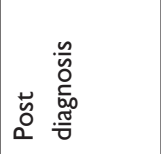 & 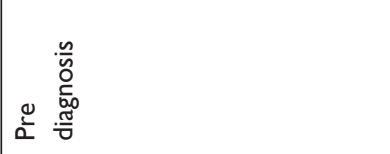 & 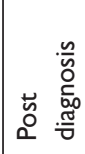 \\
\hline 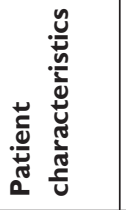 & 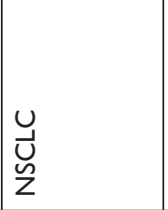 & 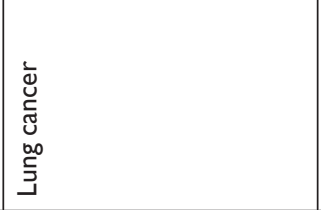 & 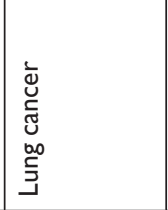 & 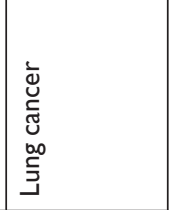 & $\begin{array}{l}u \\
\mathcal{u} \\
Z\end{array}$ & 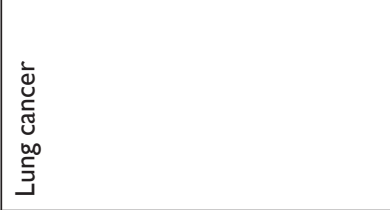 & $\begin{array}{l}u \\
u \\
u \\
z\end{array}$ \\
\hline 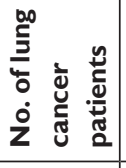 & $\stackrel{n}{\stackrel{n}{n}}$ & $\frac{\pi}{i}$ & $\begin{array}{l}\stackrel{\infty}{\infty} \\
\stackrel{m}{=}\end{array}$ & 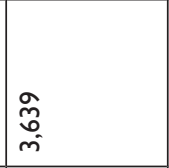 & 으 & $\frac{\stackrel{\rho}{\frac{m}{f}}}{\dot{f}}$ & $\frac{1}{\sigma}$ \\
\hline 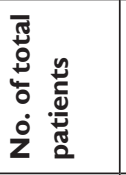 & 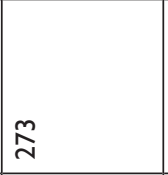 & 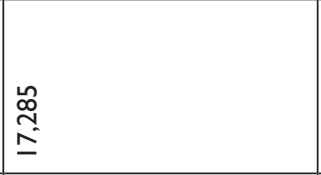 & $\stackrel{\substack{\infty \\
\stackrel{\rho}{=}}}{=}$ & 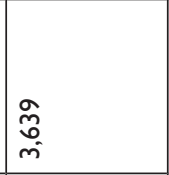 & 으 & 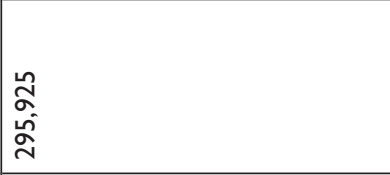 & $\frac{\pi}{\sigma}$ \\
\hline 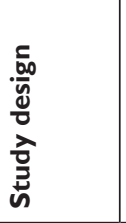 & $\begin{array}{l}\frac{\pi}{0} \\
\frac{5}{0}\end{array}$ & $\begin{array}{l}\frac{t}{0} \\
\frac{0}{0} \\
0\end{array}$ & 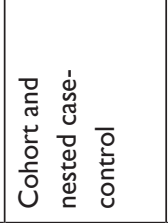 & 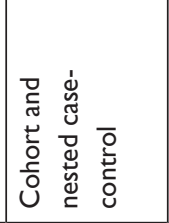 & $\begin{array}{l}\frac{5}{0} \\
\frac{0}{0} \\
0\end{array}$ & 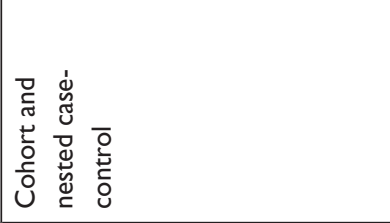 & 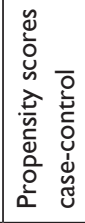 \\
\hline 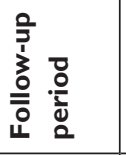 & 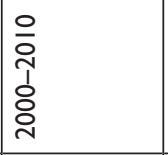 & $\begin{array}{l}\infty \\
\sigma \\
\frac{1}{1} \\
\alpha \\
\alpha\end{array}$ & 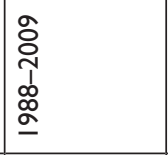 & 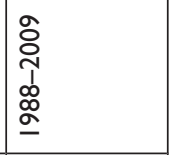 & 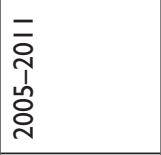 & 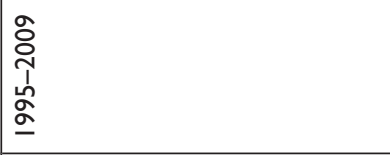 & $\begin{array}{l}\text { o } \\
\text { i } \\
\vdots \\
\sigma \\
0\end{array}$ \\
\hline نे & 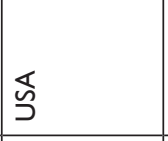 & 志 & 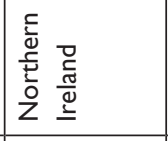 & 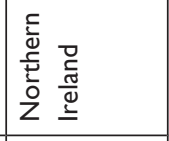 & $\begin{array}{l}\overline{\bar{g}} \\
\underline{\underline{\pi}}\end{array}$ & \begin{tabular}{|l}
$\frac{5}{\underline{n}}$ \\
$\frac{\tilde{J}}{5}$ \\
$D$
\end{tabular} & 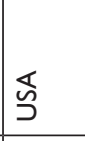 \\
\hline 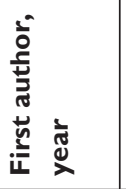 & 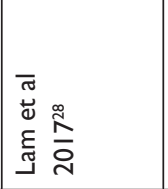 & 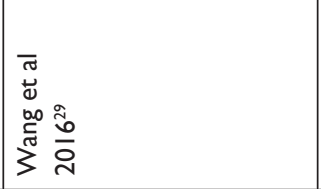 & 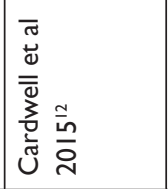 & 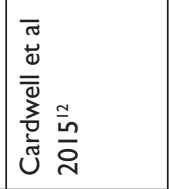 & 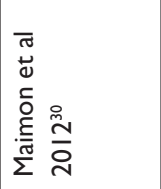 & 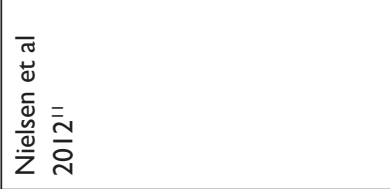 & 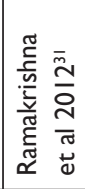 \\
\hline
\end{tabular}




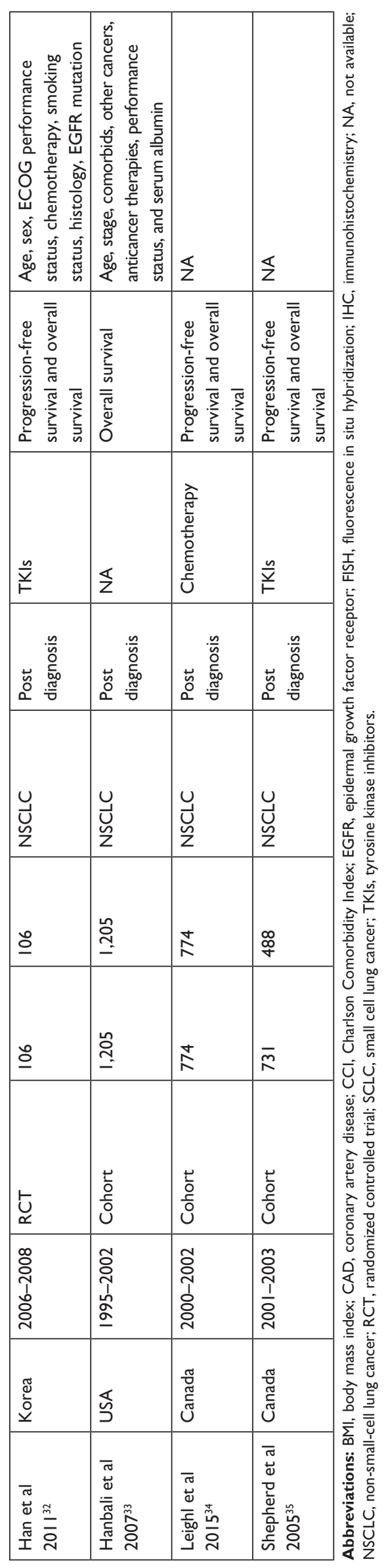

no apparent relationship between statins and progressionfree survival with low heterogeneity ( $\mathrm{HR}=0.86,95 \% \mathrm{CI}$ : $\left.0.81-0.91, I^{2}=39.9 \%, P=0.14\right)$. In three subgroup analyses, all results indicated that statin use showed no significant impact on progression-free survival (Table 5).

\section{Overall survival}

Twelve HR estimates of lung cancer overall survival were found, involving 20,712 patients (Figure 2D). ${ }^{13,23,24,26,27,30-32,34,35}$ When the relationship of statins on overall survival was analyzed, pooled HR (0.82, 95\% CI: $0.76-0.88)$ indicated an incremental overall survival of lung cancer with moderate heterogeneity $\left(I^{2}=57 \%, P=0.007\right.$; Table 6$)$. After stratification by study design, a significantly beneficial effect of overall survival was found in cohort studies ( $\mathrm{HR}=0.79,95 \%$ CI: $0.73-0.86)$ and case-control studies ( $\mathrm{HR}=0.78,95 \% \mathrm{CI}$ : $0.74-0.83$ ) but not in RCTs (HR $=1.0,95 \%$ CI: $0.87-1.14)$. In the subgroup analysis by cancer type, statins were related to the increased overall survival in NSCLC ( $\mathrm{HR}=0.78,95 \% \mathrm{CI}$ : $0.75-0.80$ ), but not in SCLC (HR $=0.86,95 \%$ CI: $0.91-1.16$ ). In addition, combined chemotherapy ( $\mathrm{HR}=0.86,95 \% \mathrm{CI}$ : $0.81-0.91$ ) and TKIs therapy (HR $=0.86,95 \%$ CI: $0.76-0.98$ ) enhanced the overall survival of patients with lung cancer.

By heterogeneity test and subgroup analysis, one study was considered as the main heterogeneity source with a negative result of overall survival. ${ }^{13}$ When we removed this study, a significant benefit of overall survival ( $\mathrm{HR}=0.79,95 \% \mathrm{CI}$ : $0.75-0.83)$ was found with low heterogeneity $\left(I^{2}=22.3 \%\right.$, $P=0.22$ ). In the subgroup analyses without this study, all the results above were not apparently changed with low heterogeneity (data not shown).

\section{Overall response rate and safety}

Three RCTs and one cohort study provided overall response rate, involving 1,127 patients..$^{13,27,30,32}$ Three studies of NSCLC with combined TKIs therapy and one study of SCLC with combined chemotherapy were found. Statin use did not show significant benefit of overall response rate with absent heterogeneity (OR $=1.08,95 \%$ CI: $0.83-1.39, I^{2}=0 \%, P=0.48$ ). When stratified by cancer type and combined therapy, the patients who took statins did not obtain better overall response rate than the patients without statins (Figure S3).

Three RCTs evaluated the safety of statin use. ${ }^{13,27,32}$ Myalgia and myositis were the main toxicities of statins. Myalgia and myositis of any grade (18\% vs $18.8 \%)$ and grade 3 or above $(0.7 \%$ vs $1.0 \%)$ were similar between the study and control groups. ${ }^{13}$ Also, statin use did not increase the adverse events of chemotherapy and TKIs therapy. In the study of statins combined with chemotherapy, almost all 
Table 2 Clinical outcome of the included studies

\begin{tabular}{|c|c|c|c|c|}
\hline First author, year & Study design & Outcome & HR $(95 \% \mathrm{CI})$ & $P$-value \\
\hline Lam et al $2017^{28}$ & Cohort & Overall survival & $0.60(0.4 I-0.89)$ & 0.011 \\
\hline Wang et al $2016^{29}$ & Cohort & Cancer-specific mortality & $1.17(0.97-1.40)$ & NA \\
\hline \multirow[t]{2}{*}{ Cardwell et al $2015^{12}$ (pre) } & Cohort & Cancer-specific mortality & $0.88(0.83-0.93)$ & $<0.001$ \\
\hline & & All-cause mortality & $0.89(0.85-0.94)$ & $<0.001$ \\
\hline \multirow[t]{3}{*}{ Cardwell et al $2015^{12}$ (post) } & Cohort & Cancer-specific mortality & $0.89(0.78-1.02)$ & 0.09 \\
\hline & & All-cause mortality & $0.91(0.80-1.02)$ & 0.1 \\
\hline & Nested case-control & Cancer-specific mortality & $0.92(0.79-1.07)$ & 0.27 \\
\hline \multirow[t]{2}{*}{ Lee et al $2017^{27}$} & RCT & Progression-free survival & $1.38(0.84-2.29)$ & 0.898 \\
\hline & & All-cause mortality & $1.03(0.58-1.80)$ & 0.466 \\
\hline \multirow[t]{2}{*}{ Han et al $201 I^{32}$} & RCT & Progression-free survival & $0.89(0.60-1.32)$ & 0.491 \\
\hline & & Overall survival & $0.88(0.57-1.35)$ & 0.491 \\
\hline \multirow[t]{2}{*}{ Seckl et al $2017^{13}$} & RCT & Progression-free survival & $0.98(0.85-1.13)$ & 0.81 \\
\hline & & Overall survival & $1.01(0.88-1.16)$ & 0.9 \\
\hline \multirow[t]{2}{*}{ Lin et al $2016^{26}$} & Propensity scores case-control & Overall survival & $0.77(0.72-0.83)$ & NA \\
\hline & Cohort & Overall survival & $0.77(0.72-0.83)$ & NA \\
\hline Huang et al $2016^{25}$ & Propensity scores case-control & All-cause mortality & $0.91(0.86-0.96)$ & $<0.01$ \\
\hline Lohinai et al $2016^{24}$ & Cohort & Overall survival & $\mathrm{I} .09(0.85-\mathrm{I} .4 \mathrm{I})$ & 0.483 \\
\hline \multirow[t]{4}{*}{ Hung et al $2017^{22}$} & Cohort & All-cause mortality & $0.58(0.54-0.62)$ & $<0.001$ \\
\hline & & Progression-free survival & NA & $<0.001$ \\
\hline & & Overall survival & NA & $<0.001$ \\
\hline & Propensity scores case-control & All-cause mortality & $0.61(0.57-0.65)$ & $<0.001$ \\
\hline \multirow[t]{2}{*}{ Leighl et al $2015^{34}$} & Cohort & Progression-free survival & $1.02(0.72-1.45)$ & 0.9 \\
\hline & & Overall survival & $0.95(0.44-1.07)$ & 0.75 \\
\hline \multirow[t]{2}{*}{ Maimon et al $2012^{30}$} & Cohort & Progression-free survival & $0.44(0.22-0.88)$ & 0.02 \\
\hline & & Overall survival & $0.63(0.36-1.09)$ & 0.01 \\
\hline \multirow[t]{2}{*}{ Hanbali et al $2007^{33}$} & Cohort & Overall survival & NA & 0.0001 \\
\hline & & All-cause mortality & $0.74(0.59-0.95)$ & 0.017 \\
\hline \multirow[t]{2}{*}{ Lee et al $2017^{23}$} & Cohort & Overall survival & $0.80(0.74-0.86)$ & $<0.001$ \\
\hline & Propensity scores case-control & Overall survival & $0.83(0.73-0.95)$ & 0.007 \\
\hline Ramakrishna et al $2012^{3 !}$ & Cohort & Overall survival & $0.66(0.45-0.96)$ & 0.03 \\
\hline \multirow[t]{2}{*}{ Shepherd et al $2005^{35}$} & Cohort & Progression-free survival & $0.72(0.42-1.23)$ & 0.2 \\
\hline & & Overall survival & $0.82(0.5 \mathrm{I}-1.34)$ & 0.43 \\
\hline \multirow[t]{2}{*}{ Nielsen et al $2012^{\prime \prime}$} & Cohort & Cancer-specific mortality & $0.87(0.83-0.93)$ & $<0.001$ \\
\hline & Nested case-control & Cancer-specific mortality & $0.83(0.79-0.88)$ & $<0.001$ \\
\hline
\end{tabular}

Abbreviations: NA, not available; RCT, randomized controlled trial.

grade $3-5$ adverse events $(81.2 \%$ vs $81.4 \%, P=0.94)$ were similar between the pravastatin and placebo arms. ${ }^{13}$ In two studies of statins combined with TKIs, the incidence of grade 3 or above skin rash was low and similar (3/88 vs 2/86) between the statins and placebo groups. ${ }^{27,32}$

\section{Publication bias}

The results of Egger's tests $(P>0.1)$ and Begg's tests $(P>0.1)$ showed that publication bias was absent in all analyses of all-cause mortality, overall survival, and progression-free survival (Figure 3). For cancer-specific mortality, slight publication bias was found in the analysis using Egger's tests $(P=0.082)$ and Begg's tests $(P=0.035$; Figure 3$)$. After a correction for possible publication bias through the trim-andfill method, cancer-specific mortality ( $\mathrm{HR}=0.86,95 \% \mathrm{CI}$ : $0.81-0.92$ ) remained to significantly benefit from statin use.

\section{Discussion}

Our study not only investigated the relationship between statins and lung cancer mortality and survival but also assessed 


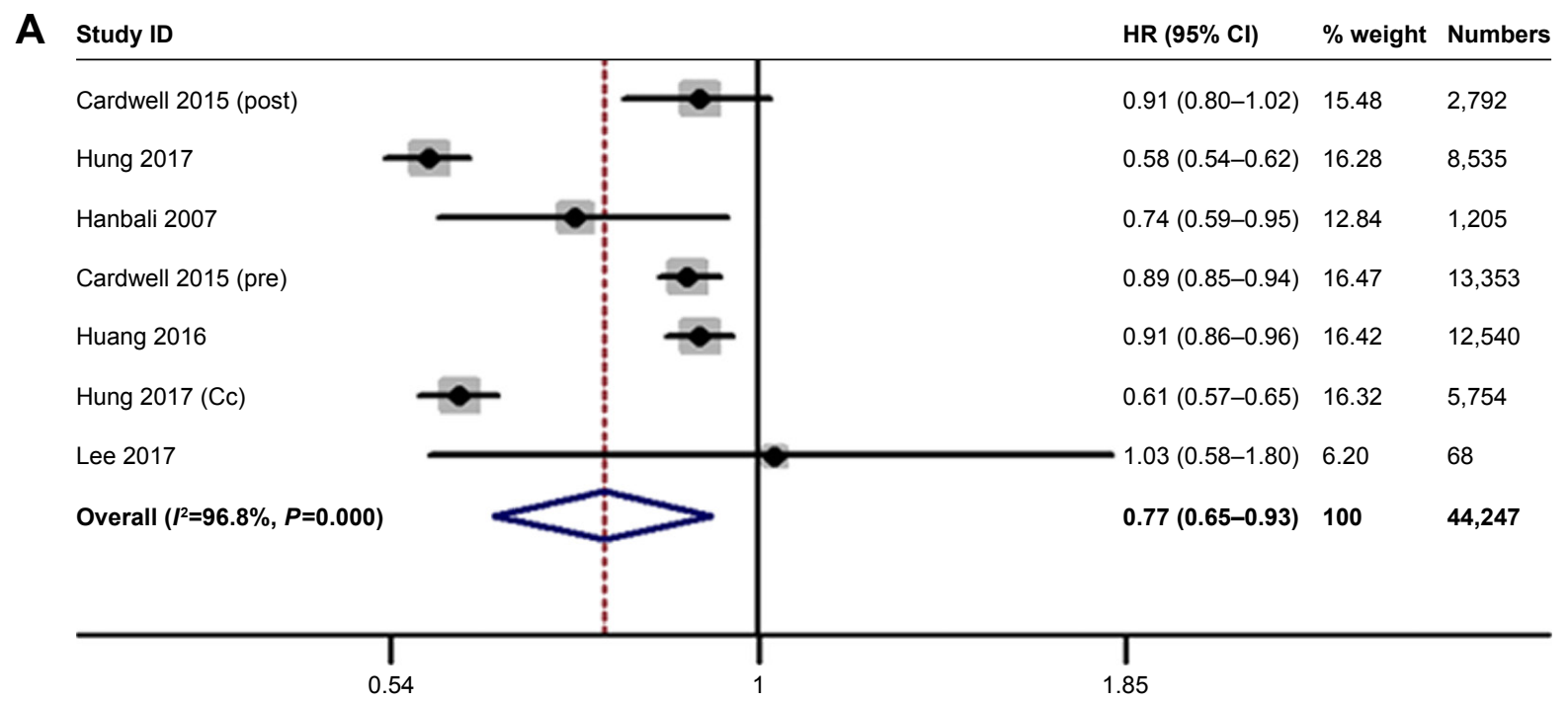

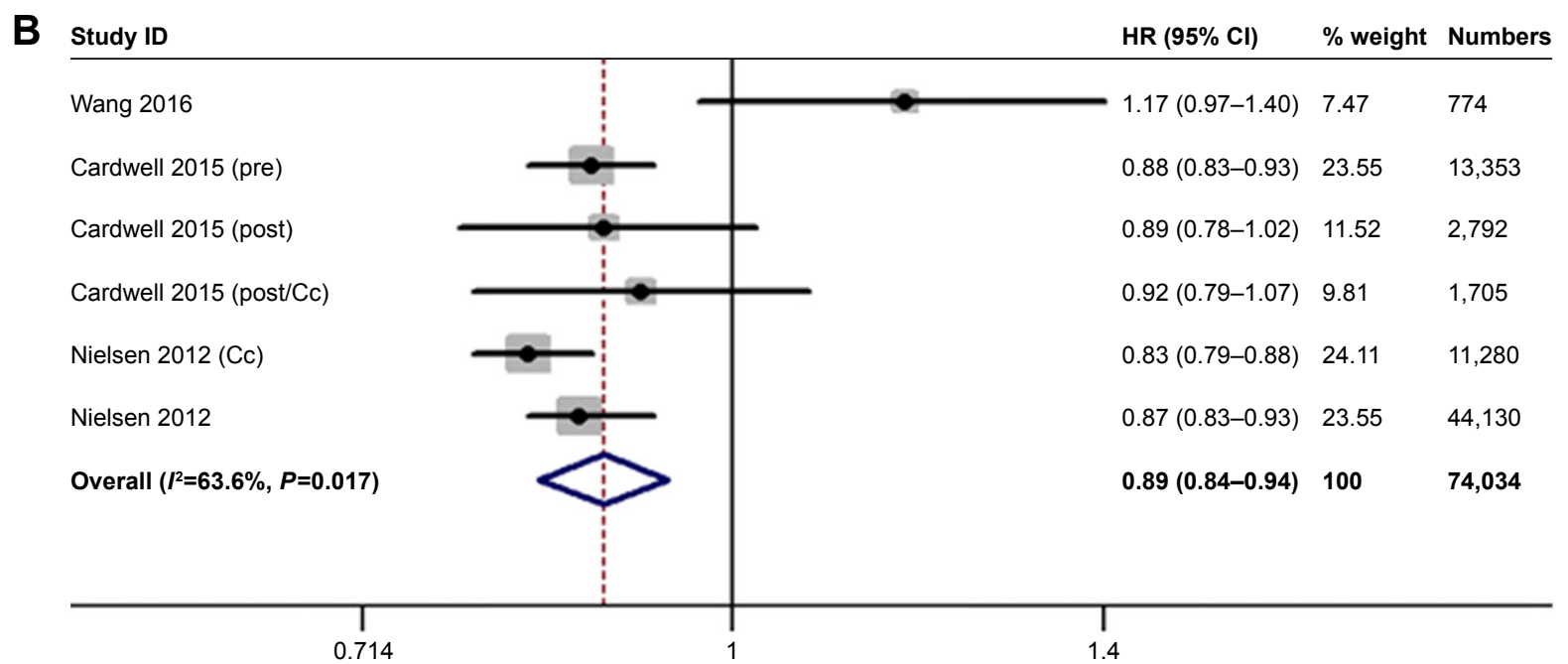

\begin{tabular}{|c|c|c|c|}
\hline Study ID & HR $(95 \% \mathrm{Cl})$ & $\%$ weight & Numbers \\
\hline Leighl 2005 & $1.02(0.72-1.45)$ & 18.63 & 774 \\
\hline Shepherd 2005 & $0.72(0.42-1.23)$ & 10.38 & 488 \\
\hline Han 2011 & $0.89(0.60-1.32)$ & 16.10 & 106 \\
\hline Maimon 2012 & $0.44(0.22-0.88)$ & 6.87 & 107 \\
\hline Lee 2017 & $1.38(0.84-2.29)$ & 11.52 & 68 \\
\hline Seckl 2017 & $0.98(0.85-1.13)$ & 36.51 & 846 \\
\hline Overall $\left(I^{2}=39.9 \%, P=0.140\right)$ & $0.93(0.76-1.13)$ & 100 & 2,389 \\
\hline
\end{tabular}

Figure 2 (Continued) 


\begin{tabular}{l} 
D Study ID \\
\hline Seckl 2017 \\
Lee 2017 \\
Lee 2017 (Cc)
\end{tabular}

Figure 2 Forest plot: overall meta-analysis of mortality and survival between statin use and lung cancer.

Notes: (A) all-cause mortality; (B) cancer-specific mortality; (C) progression-free survival; and (D) overall survival. Weights are from random-effects analysis. Abbreviation: Cc, case-control.

the overall response rate and safety of statins. The summary results involving 98,445 lung cancer patients suggested that statin use was associated with the benefit of all-cause mortality, cancer-specific mortality, and overall survival. However, statin use did not affect the progression-free survival of patients with lung cancer. Although these results had significant heterogeneity, the effect of statin use was not materially altered when the main heterogeneity source was removed. Meanwhile, we performed some subgroup analyses to identify sources of these significant heterogeneity, including prediagnosis vs postdiagnosis use, NSCLC vs SCLC, and TKIs vs chemotherapy. Both prediagnosis and postdiagnosis statin use decreased the risk of all-cause mortality and cancer-specific mortality and increased overall survival, but exerted no impact on progression-free survival. Statin use seemingly increased the benefit of chemotherapy and TKIs therapy on overall survival and did not enhance their toxicities. Our study also indicated that the beneficial effect of statin use in terms of overall survival was seen mainly in NSCLC patients but not in SCLC patients. Statins in treating lung cancer had no significant impact on the progression-free survival, whether in NSCLC patients or in SCLC patients (Table 5).

Subgroup analysis based on study design showed that the potential beneficial association between statin use and

Table 3 HR estimates of all-cause mortality between statins and lung cancer

\begin{tabular}{|c|c|c|c|c|}
\hline & No. of reports & Pooled HR $(95 \% \mathrm{Cl})$ & $I^{2}(\%)$ & $P$ for heterogeneity \\
\hline Overall estimation & 7 & $0.77(0.65-0.93)$ & 96.80 & $<0.001$ \\
\hline \multicolumn{5}{|l|}{ Subgroup analysis } \\
\hline \multicolumn{5}{|l|}{ Study design } \\
\hline Cohort & 4 & $0.77(0.59-0.99)$ & 97.10 & $<0.001$ \\
\hline Case control & 2 & $0.75(0.50-1.10)$ & 98.80 & $<0.001$ \\
\hline $\mathrm{RCT}$ & I & $\mathrm{I} .03(0.58-1.8 \mathrm{I})$ & & \\
\hline \multicolumn{5}{|l|}{ Timing of statin use } \\
\hline Postdiagnosis & 5 & $0.7 \mathrm{I}(0.59-0.85)$ & 91.30 & $<0.001$ \\
\hline Prediagnosis & 2 & $0.90(0.87-0.93)$ & 0 & 0.559 \\
\hline \multicolumn{5}{|c|}{ Combined treatment } \\
\hline TKIs & 3 & $0.60(0.55-0.66)$ & 74.30 & 0.048 \\
\hline Chemotherapy & 3 & $0.63(0.57-0.7 I)$ & 31.20 & 0.234 \\
\hline
\end{tabular}

Abbreviations: RCT, randomized controlled trial; TKIs, tyrosine kinase inhibitors. 
Table 4 HR estimates of cancer-specific mortality between statins and lung cancer

\begin{tabular}{|c|c|c|c|c|}
\hline & No. of reports & Pooled HR (95\% CI) & $I^{2}(\%)$ & $P$ for heterogeneity \\
\hline Overall estimation & 6 & $0.89(0.84-0.94)$ & 63.60 & $<0.001$ \\
\hline \multicolumn{5}{|l|}{ Subgroup analysis } \\
\hline \multicolumn{5}{|l|}{ Study design } \\
\hline Cohort & 4 & $0.91(0.84-0.99)$ & 67.60 & 0.026 \\
\hline Case control & 2 & $0.85(0.78-0.93)$ & 36.30 & 0.21 \\
\hline \multicolumn{5}{|l|}{ Timing of statin use } \\
\hline Prediagnosis & 3 & $0.7 \mathrm{I}(0.59-0.85)$ & 91.30 & $<0.001$ \\
\hline Postdiagnosis & 3 & $0.90(0.87-0.93)$ & 0 & 0.559 \\
\hline
\end{tabular}

survival in lung cancer patients can be observed in only observational studies but not in RCTs. One possible explanation was that these existing observational studies harbored some selective bias and confounding factors, which led to some differences in the results between observational studies and RCTs. Given their low selective bias and few confounding factors, RCTs were regarded as the gold standard for evaluating most treatments with the most scientifically sound method. Another possible explanation was that the present RCTs possessed the following limitations. First, the pooled HR involved only three RCTs, including two small-sample open-label RCTs of advanced NSCLC and one multi-center double-blind RCT of SCLC. ${ }^{13,27,32}$ The results were often unstable and were of low inspection efficiency because of the small size of studies and patients. Some real differences may not be discovered due to the low power of a smallsample study. Second, two open-label RCTs used simvastatin combined TKIs to treat the patients with advanced NSCLC after failure of platinum-based chemotherapy. ${ }^{27,32}$ Patients with advanced NSCLC had a relatively short survival time and received short-term statin use, which might affect the efficacy of statins. A cohort study showed that the patients who received postdiagnosis statins with $\geq 12$ prescriptions had a significantly low risk of cancer-specific mortality (adjusted HR $=0.81,95 \%$ CI: 0.67-0.98) and all-cause mortality (adjusted HR $=0.83,95 \%$ CI: $0.70-0.97$ ). However, this result was not observed in patients who received postdiagnosis statins with $\leq 11$ prescriptions (cancer-specific mortality, adjusted HR $=0.94,95 \%$ CI: $0.81-1.09$ and allcause mortality, adjusted HR $=0.95,95 \%$ CI: 0.83-1.09). ${ }^{12}$ Five people evaluated the therapeutic value of statin use in patients with stage IV lung cancer. They found that combination with statins did not significantly benefit the overall survival of patients $(\mathrm{HR}=0.87,95 \% \mathrm{CI}$ : $0.75-1.03) .{ }^{26}$ However, another cohort study suggested that the patients who received statins and TKIs had a longer progression-free survival and overall survival than the patients who received only TKIs. ${ }^{22}$ In addition, statins were related to a reduction of all-cause mortality $(\mathrm{HR}=0.58,95 \% \mathrm{CI}: 0.54-0.62) .{ }^{22}$ The largest difference between the two aforementioned studies was that only patients with stage IV lung cancer were included in the first study. 22,26 Therefore, a high-quality RCT must be conducted to evaluate the effect of TKIs and long-term statin use on early NSCLC. Finally, only one multi-center,

Table 5 HR estimates of progression-free survival between statins and lung cancer

\begin{tabular}{|c|c|c|c|c|}
\hline & No. of reports & Pooled HR (95\% Cl) & $I^{2}(\%)$ & $P$ for heterogeneity \\
\hline Overall estimation & 6 & $0.93(0.76-1.13)$ & 39.90 & 0.14 \\
\hline \multicolumn{5}{|l|}{ Subgroup analysis } \\
\hline \multicolumn{5}{|l|}{ Study design } \\
\hline Cohort & 3 & $0.74(0.47-1.17)$ & 58.50 & 0.29 \\
\hline RCT & 3 & $0.99(0.87-1.13)$ & 0 & 0.37 \\
\hline \multicolumn{5}{|l|}{ Cancer type } \\
\hline NSCLC & 5 & $0.88(0.65-1.19)$ & 50.10 & 0.093 \\
\hline SCLC & I & $0.98(0.85-1.13)$ & & \\
\hline \multicolumn{5}{|l|}{ Combined treatment } \\
\hline TKIs & 3 & $0.85(0.49-1.49)$ & 70.90 & 0.032 \\
\hline Chemotherapy & 2 & $0.99(0.86-1.12)$ & 0.00 & 0.836 \\
\hline
\end{tabular}

Abbreviations: NSCLC, non-small-cell lung cancer; RCT, randomized controlled trial; SCLC, small cell lung cancer; TKIs, tyrosine kinase inhibitors. 
Table 6 HR estimates of overall survival between statins and lung cancer

\begin{tabular}{|c|c|c|c|c|}
\hline & No. of reports & Pooled HR (95\% CI) & $I^{2}(\%)$ & P for heterogeneity \\
\hline Overall estimation & 12 & $0.82(0.76-0.88)$ & 57.00 & 0.007 \\
\hline \multicolumn{5}{|l|}{ Study design } \\
\hline Cohort & 8 & $0.79(0.73-0.86)$ & 40.70 & 0.107 \\
\hline Case control & 2 & $0.78(0.74-0.83)$ & 0.00 & 0.326 \\
\hline RCT & 2 & $1.0(0.87-1.14)$ & 0 & 0.055 \\
\hline \multicolumn{5}{|l|}{ Cancer type } \\
\hline NSCLC & 10 & $0.78(0.75-0.80)$ & 0.00 & 0.753 \\
\hline SCLC & 2 & $1.03(0.91-1.16)$ & 0.00 & 0.604 \\
\hline \multicolumn{5}{|l|}{ Combined treatment } \\
\hline TKls & 4 & $0.86(0.76-0.98)$ & 74.30 & 0.716 \\
\hline Chemotherapy & 5 & $0.86(0.8 I-0.9 I)$ & 54.60 & 0.061 \\
\hline
\end{tabular}

Abbreviations: NSCLC, non-small-cell lung cancer; RCT, randomized controlled trial; SCLC, small cell lung cancer; TKIs, tyrosine kinase inhibitors.

double-blind RCT used pravastatin combined with first-line standard chemotherapy to treat SCLC. ${ }^{13}$ Compared with chemotherapy alone, pravastatin and chemotherapy did not significantly prolong the progression-free survival and overall survival of patients with SCLC. This study has some limitations. Pravastatin, as a hydrophilic statin, exerted no beneficial effects on cancer prevention or cancer death as observed in a trial of patients with coronary artery disease. ${ }^{36}$

\section{A Begg's funnel plot with pseudo 95\% confidence limits}
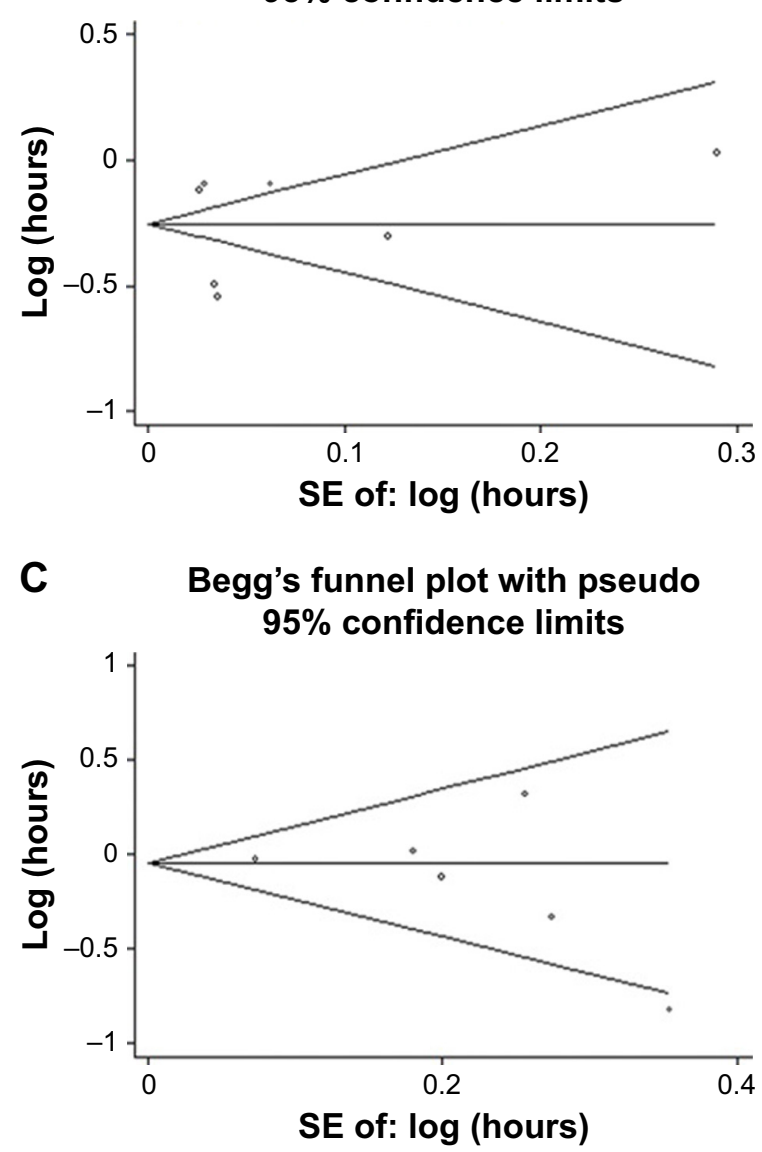

\section{B Begg's funnel plot with pseudo} $95 \%$ confidence limits
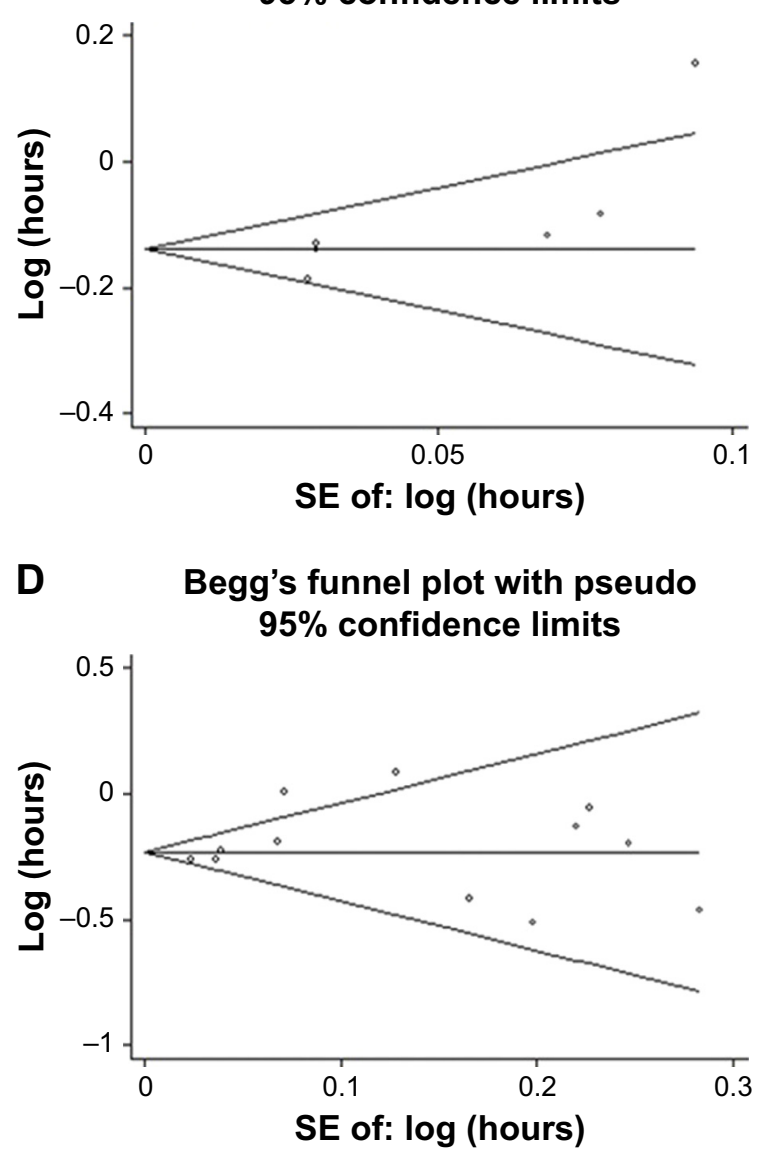

Figure 3 Funnel plot for publication bias of mortality and survival between statin use and lung cancer.

Note: $(\mathbf{A})$ all cause mortality (Egger's test $=0.848$ and Begg's test $=0.548)$; $(\mathbf{B})$ cancer-specific mortality (Egger's test $=0.082$ and Begg's test $=0.035)$; $(\mathbf{C})$ progression-free survival (Egger's test $=0.43 \mathrm{I}$ and Begg's test $=0.133$ ); and $(\mathbf{D})$ overall survival (Egger's test $=0.425$ and Begg's test $=0.732$ ). 
In a cohort study of lung cancer, postdiagnosis pravastatin had no significant association with cancer-specific mortality (adjusted HR $=1.23,95 \% \mathrm{CI}: 0.85-1.77) .{ }^{12}$ Simvastatin, as a lipophilic statin, demonstrated more benefits than pravastatin for patients with lung cancer in vivo and vitro. ${ }^{9,12}$ Postdiagnosis simvastatin was associated with the reduction of cancer-specific mortality (adjusted HR $=0.92,95 \%$ CI: 0.86-0.97) and all-cause mortality (adjusted HR $=0.92$, 95\% CI: 0.87-0.97). ${ }^{12}$ A cohort study showed that lipophilic statins exerted greater beneficial effects on overall survival (HR $=0.92$, 95\% CI: 0.88-0.96) and lung cancer survival (HR $=0.91,95 \%$ CI: $0.87-0.96)$ than hydrophilic statins. ${ }^{26}$ In addition, we found that statin use seemingly brought about more benefit in overall survival for NSCLC ( $\mathrm{HR}=0.78$, 95\% CI: 0.75-0.80) compared with SCLC (HR =1.03, 95\% CI: 0.91-1.16). Therefore, additional high-quality RCTs are needed to evaluate the effect of simvastatin and chemotherapy for NSCLC patients.

In our study, statin use showed the benefit of mortality and overall survival for lung cancer, but no impact on progression-free survival. One hypothesis was that the benefit of statin use could be attributed to the role of statins' value on cardiocerebrovascular risk reduction. Patients with lung cancer usually have a large burden of COPD and cardiocerebrovascular disease owing to the shared risk factor of smoking. However, similar HR estimates of all-cause mortality were found in patients with lung cancer with or without comorbidities (Figure S2). Meanwhile, statin use was confirmed to be associated with the risk reduction of cancerspecific mortality. Therefore, whether and why statins had no impact on progression-free survival remains to be further observed and studied in clinical practice.

Our results suggested that statins combined with chemotherapy and TKIs were associated with lower all-cause mortality and better overall survival of lung cancer patients compared with patients without statin use (Tables 3 and 6). Already, statins have been found to affect lung cancer mortality and overall survival through multiple mechanisms. In in vitro studies, simvastatin was found to induce cell cycle arrest or potential apoptosis in lung cancer cells. ${ }^{37,38}$ Simvastatin could reverse resistance to TKIs therapy for NSCLC cell lines with T790M mutation by downregulating an $\mathrm{AKT} / \beta$-catenin signaling-dependent pathway. ${ }^{9}$ In in vivo studies, the combination of TKIs with statins showed better overall response rate and progression-free survival of patients with NSCLC harboring KRAS mutations compared with those without KRAS mutation. ${ }^{10}$ The potential mechanism was that statins decreased the expression of the RAS protein, which resulted in the inhibition of the RAF/ERK and AKT pathways and overcame TKIs resistance. In addition, atorvastatin was confirmed to carboplatin resistance in human NCSLC through suppression of AKT activation and upregulation of TIMP- $1 .{ }^{8}$ Our meta-analysis had several strengths. To the best of our knowledge, this study was the most comprehensive meta-analysis assessing the relationship among lung cancer mortality, survival, and statin use, which included English original articles and meeting abstracts. Two meta-analyses only assessed that statins did not decrease the risk of developing lung cancer. ${ }^{39,40}$ Many previous papers investigated the relationship between statins and cancer. ${ }^{15-18}$ However, most studies evaluated only the survival benefit or mortality of cancer patients who received statin treatment. We not only simultaneously assessed the effect of statin use on lung cancer mortality and survival but also evaluated the safety of statins and the overall response rate of statins for lung cancer. In addition, we performed many subgroup analyses to obtain detailed data about the relationship between statins and lung cancer. Meanwhile, the robustness of the results was confirmed through many methods, including heterogeneity test, subgroup analysis, and publication bias analysis.

Certainly, our meta-analysis had some potential limitations. First, most included studies were cohort studies, which were deemed to confer incoherent shortcomings. Second, the language of these included studies was limited to English, which likely leads to selection bias. Third, some main results had high heterogeneity and publication bias. Fourth, some patients simultaneously received prediagnosis and postdiagnosis statin treatment in some cohort studies. The authors did not assess the effect of postdiagnosis statins on the association between lung cancer mortality and prediagnosis statins. ${ }^{12}$ Fifth, with only few included studies that did not perform adjustment for important risk factors, unadjusted factors relative to statins possibly affected the final results of individual studies. Sixth, only two studies provided the dose-response analyses. ${ }^{12,22}$ Therefore, the dose-response analysis should be interpreted with caution.

\section{Conclusion}

This meta-analysis showed that statin use for lung cancer patients was potentially associated with the decreasing risk of mortality and the improvement of overall survival in observational studies, which was not supported by existing evidence from RCTs. Statin use did not significantly influence the progression-free survival and overall response rate of lung cancer patients. Statins were safe and did not increase the toxicity of chemotherapy and TKIs. Long-term use of 
statins, mainly including lipophilic statins, may potentially bring about the beneficial effect of mortality and overall survival for patients with early stage NSCLC. It is reasonable to believe that statins at least do not appear to be harmful and may be beneficial for NSCLC patients. Additional high-quality RCTs investigating the relationship between long-term statin use and survival from early stage NSCLC are warranted in the future.

\section{Acknowledgment}

Dao-Kui Xia, Zhi-Gang Hu, and Yu-Feng Tian are co-first authors for this study.

\section{Disclosure}

The authors report no conflicts of interests in this work.

\section{References}

1. Chen W, Zheng R, Baade PD, et al. Cancer statistics in China, 2015. CA Cancer J Clin. 2016;66(2):115-132.

2. Cholesterol Treatment Trialists' (CTT) Collaborators, Mihaylova B, Emberson J, et al. The effects of lowering LDL cholesterol with statin therapy in people at low risk of vascular disease: meta-analysis of individual data from 27 randomized trials. Lancet. 2012;380(9841): 581-590.

3. Mills EJ, Rachlis B, Wu P, Devereaux PJ, Arora P, Perri D. Primary prevention of cardiovascular mortality and events with statin treatments: a network meta-analysis involving more than 65,000 patients. $\mathrm{J} \mathrm{Am} \mathrm{Coll}$ Cardiol. 2008;52(22):1769-1781.

4. Hwang KE, Na KS, Park DS, et al. Apoptotic induction by simvastatin in human lung cancer A549 cells via Akt signaling dependent downregulation of survivin. Invest New Drugs. 2011;29(5):945-952.

5. Liu H, Wang Z, Li Y, Li W, Chen Y. Simvastatin prevents proliferation and bone metastases of lung adenocarcinoma in vitro and in vivo. Neoplasma. 2013;60(03):240-246.

6. Hanai J, Doro N, Sasaki AT, et al. Inhibition of lung cancer growth: ATP citrate lyase knockdown and statin treatment leads to dual blockade of mitogen-activated protein kinase (MAPK) and phosphatidylinositol3-kinase (PI3K)/AKT pathways. J Cell Physiol. 2012;227(4): 1709-1720.

7. Chen J, Liu B, Yuan J, et al. Atorvastatin reduces vascular endothelial growth factor (VEGF) expression in human non-small cell lung carcinomas (NSCLCs) via inhibition of reactive oxygen species (ROS) production. Mol Oncol. 2012;6(1):62-72.

8. Chen J, Lan T, Hou J, et al. Atorvastatin sensitizes human non-small cell lung carcinomas to carboplatin via suppression of AKT activation and upregulation of TIMP-1. Int J Biochem Cell Biol. 2012;44(5): 759-769.

9. Hwang KE, Kwon SJ, Kim YS, et al. Effect of simvastatin on the resistance to EGFR tyrosine kinase inhibitors in a non-small cell lung cancer with the T790M mutation of EGFR. Exp Cell Res. 2014;323(2): 288-296.

10. Fiala O, Pesek M, Finek J, et al. Statins augment efficacy of EGFR-TKIs in patients with advanced-stage non-small cell lung cancer harbouring KRAS mutation. Tumour Biol. 2015;36(8):5801-5805.

11. Nielsen SF, Nordestgaard BG, Bojesen SE. Statin use and reduced cancer-related mortality. N Engl J Med. 2012;367(19):1792-1802.

12. Cardwell CR, McMenamin U, Hughes CM, Murray LJ. Statin use and survival from lung cancer: a population-based cohort study. Cancer Epidemiol Biomarkers Prev. 2015;24(5):833-841.
13. Seckl MJ, Ottensmeier CH, Cullen M, et al. Multicenter, phase III, randomized, double-blind, placebo-controlled trial of pravastatin added to first-line standard chemotherapy in small-cell lung cancer (LUNGSTAR). J Clin Oncol. 2017;35(14):1506-1514.

14. Manthravadi S, Shrestha A, Madhusudhana S. Impact of statin use on cancer recurrence and mortality in breast cancer: a systematic review and meta-analysis. Int J Cancer. 2016;139(6):1281-1288.

15. Li X, Wu XB, Chen Q. Statin use is not associated with reduced risk of skin cancer: a meta-analysis. Br J Cancer. 2014;110(3):802-807.

16. Nayan M, Punjani N, Juurlink DN, et al. Statin use and kidney cancer survival outcomes: a systematic review and meta-analysis. Cancer Treat Rev. 2017;52:105-116.

17. Gray RT, Coleman HG, Hughes C, Murray LJ, Cardwell CR. Statin use and survival in colorectal cancer: results from a population-based cohort study and an updated systematic review and meta-analysis. Cancer Epidemiol. 2016;45:71-81.

18. Wells G, Shea B, O'Connell D. The Newcastle-Ottawa Scale (NOS) for assessing the quality of nonrandomized studies in meta-analyses; 2010. Available from: www.ohri.ca/programs/clinical_epidemiology/ oxford_web.ppt. Accessed November 25, 2017.

19. Higgins J, Green S, editors. Cochrane Handbook for Systematic Reviews of Interventions Version 5.1.0 [Updated March 2011]. The Cochrane Collaboration; 2011. Available at: http://handbook-5-1.cochrane.org. Accessed March 16, 2015.

20. Viechtbauer W. Bias and efficiency of meta-analytic variance estimators in the random-effects model. J Educ Behav Stat. 2005;30(3):261-293.

21. Raudenbush SW. Analyzing effect sizes: random-effects models. In: Cooper H, Hedges LV, Valentine JC, editors. The Handbook of Research Synthesis and Meta-analysis. 2nd ed. New York: Sage; 2009; 295-316.

22. Hung MS, Chen IC, Lee CP, et al. Statin improves survival in patients with EGFR-TKI lung cancer: a nationwide population-based study. PLoS One. 2017;12(2):e0171137.

23. Lee YG, Lee JH, Jang JS, Kim JH. Prognostic benefit of statin with or without metformin in elderly patients with advanced non-small cell lung cancer: a nationwide population-based outcome study. J Clin Oncol. 2017;35(15_suppl):e20661.

24. Lohinai Z, Dome P, Szilagyi Z, et al. From bench to bedside: attempt to evaluate repositioning of drugs in the treatment of metastatic small cell lung cancer (SCLC). PLoS One. 2016;11(1):e0144797.

25. Huang WY, Li CH, Lin CL, Liang JA. Long-term statin use in patients with lung cancer and dyslipidemia reduces the risk of death. Oncotarget. 2016;7(27):42208-42214.

26. Lin JJ, Ezer N, Sigel K, Mhango G, Wisnivesky JP. The effect of statins on survival in patients with stage IV lung cancer. Lung Cancer. 2016;99: 137-142.

27. Lee Y, Lee KH, Lee GK, et al. Randomized phase II study of afatinib plus simvastatin versus afatinib alone in previously treated patients with advanced nonadenocarcinomatous non-small cell lung cancer. Cancer Res Treat. 2017;49(4):1001-1011.

28. Lam VK, Bentzen SM, Mohindra P, et al. Obesity is associated with long-term improved survival in definitively treated locally advanced non-small cell lung cancer (NSCLC). Lung Cancer. 2017;104:52-57.

29. Wang A, Aragaki AK, Tang JY, et al. Statin use and all-cancer survival: prospective results from the Women's Health Initiative. Br J Cancer. 2016;115(1):129-135.

30. Maimon N, Keizman D. Gottfried M. Statins (ASIs) may improve the outcome of erlotinib as second line treatment (tx) in patients (pts) with metastatic non-small cell lung cancer (mNSCLC). J Clin Oncol. 2012;30(15_Suppl):e18108.

31. Ramakrishna S, Andrei AC, Varlotto J, et al. Statin use is associated with decreased local recurrence and improved overall survival in resectable non-small cell lung cancer (NSCLC). Chest. 2012;142(4):925A-925B.

32. Han JY, Lee SH, Yoo NJ, et al. A randomized phase II study of gefitinib plus simvastatin versus gefitinib alone in previously treated patients with advanced non-small cell lung cancer. Clin Cancer Res. 2011; 17(6):1553-1560. 
33. Hanbali AS, Wang D, Jankowski M. The use of statins may prolong survival of non-small cell lung cancer patients: final update from 1,205 cases. J Clin Oncol. 2007;(18_suppl):7638.

34. Leighl NB, Paz-Ares L, Douillard JY. Randomized phase III study of matrix metalloproteinase inhibitor BMS-275291 in combination with paclitaxel and carboplatin in advanced non-small-cell lung cancer: National Cancer Institute of Canada-Clinical Trials Group Study BR. 18. J Clin Oncol. 2015;23(12):2831-2839.

35. Shepherd FA, Rodrigues Pereira J, Ciuleanu T, et al. Erlotinib in previously treated non-small-cell lung cancer. N Engl J Med. 2005;353(2): 123-132.

36. Hague WE, Simes J, Kirby A, et al. Long-term effectiveness and safety of pravastatin in patients with coronary heart disease: sixteen years of follow-up of the LIPID study. Circulation. 2016;133(11):1851-1860.
37. Liang YW, Chang CC, Hung CM, Chen TY, Huang TY, Hsu YC. Preclinical activity of simvastatin induces cell cycle arrest in G1 via blockade of cyclin D-Cdk4 expression in non-small cell lung cancer (NSCLC). Int J Mol Sci. 2013;14(3):5806-5816.

38. Yu X, Pan Y, Ma H, Li W. Simvastatin inhibits proliferation and induces apoptosis in human lung cancer cells. Oncol Res. 2013;20(8):351-357.

39. Wang J, Li C, Tao H, et al. Statin use and risk of lung cancer: a metaanalysis of observational studies and randomized controlled trials. PLoS One. 2013;8(10):e77950.

40. Tan M, Song X, Zhang G, et al. Statins and the risk of lung cancer: a meta-analysis. PLoS One. 2013;8(2):e57349. 


\section{Supplementary materials}

Table SI Methodological quality of cohort study and case-control study using the Newcastle-Ottawa scale

\begin{tabular}{|l|l|l|l|l|}
\hline First author, year & Selection & Comparability & Exposure/outcome & Total score \\
\hline Hung et al $2017^{\prime}$ & 4 & 2 & 3 & 9 \\
\hline Lee et al $2017^{2}$ & 3 & 2 & 3 & 8 \\
\hline Huang et al $2016^{3}$ & 3 & 2 & 2 & 7 \\
\hline Lin et al 2016 & 2 & 2 & 2 & 6 \\
\hline Lohinai et al $2016^{5}$ & 2 & 1 & 2 & 5 \\
\hline Wang et al $2016^{6}$ & 3 & 2 & 2 & 8 \\
\hline Lam et al $2017^{7}$ & 4 & 2 & 3 & 9 \\
\hline Cardwell et al $2015^{8}$ (pre) & 4 & 2 & 3 & 9 \\
\hline Cardwell et al $2015^{8}$ (post) & 4 & 2 & 3 & 9 \\
\hline Maimon et al $2012^{9}$ & 2 & 2 & 2 & 6 \\
\hline Nielsen et al $2012^{10}$ & 4 & 2 & 3 & 9 \\
\hline Ramakrishna et al $2012^{11}$ & 2 & 0 & 2 & 4 \\
\hline Hanbali et al $2007^{12}$ & 2 & 2 & 2 & 6 \\
\hline Leighl 2005 & 2 & 0 & 2 & 4 \\
\hline Shepherd et al 2005 & 2 & 0 & 2 & 4 \\
\hline
\end{tabular}

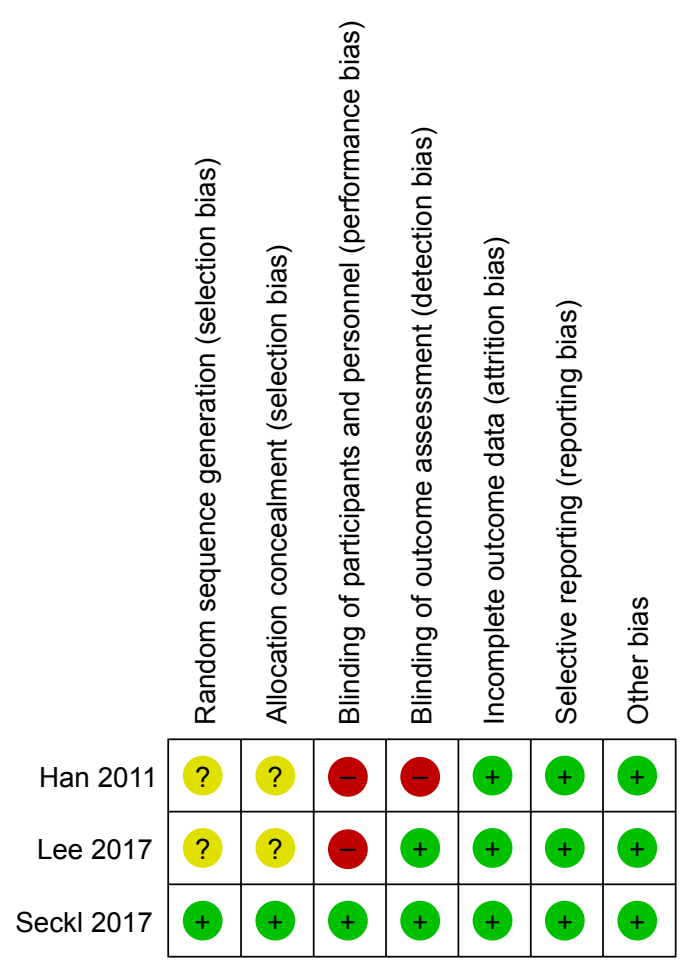

Figure SI Methodological quality of included randomized controlled trials. 


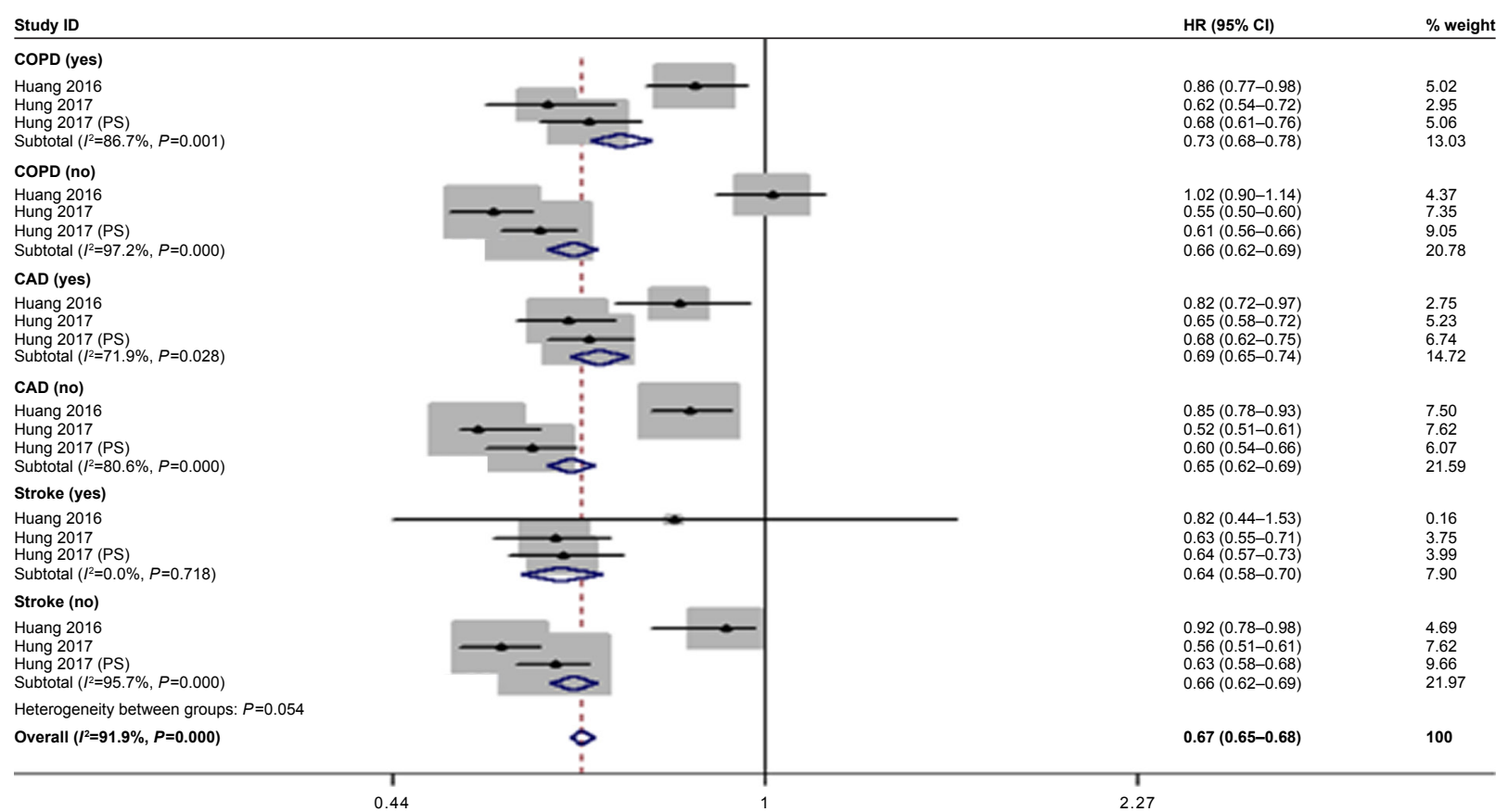

Figure S2 Forest plot: overall meta-analysis of all-cause mortality between statin use and lung cancer with or without comorbidities. Abbreviation: CAD, coronary artery disease.

\begin{tabular}{|c|c|c|}
\hline Study ID & OR $(95 \% \mathrm{Cl})$ & $\%$ weight \\
\hline \multicolumn{3}{|l|}{ NSCLC } \\
\hline Lee $2017 \quad \leftarrow$ & $0.57(0.09-3.64)$ & 2.66 \\
\hline Han 2011 & $1.36(0.61-3.03)$ & 9.09 \\
\hline Maimon 2012 & $1.75(0.78-3.91)$ & 7.94 \\
\hline Subtotal $\left(I^{2}=0.0 \%, P=0.548\right)$ & $1.41(0.82-2.42)$ & 19.68 \\
\hline \multicolumn{3}{|l|}{ SCLC } \\
\hline Seckl 2017 & $0.99(0.74-1.33)$ & 80.32 \\
\hline Subtotal $\left(I^{2}=. \%, P=.\right)$ & $0.99(0.74-1.33)$ & 80.32 \\
\hline Overall $\left(I^{2}=0.0 \%, P=0.480\right)$ & $1.08(0.83-1.39)$ & 100 \\
\hline $\begin{array}{c}1 \\
0.0888\end{array}$ & $\begin{array}{c}7 \\
11.3\end{array}$ & \\
\hline
\end{tabular}

Figure S3 Forest plot: overall meta-analysis of overall response rate between statin use and lung cancer.

Abbreviations: NSCLC, non-small-cell lung cancer; SCLC, small cell lung cancer.

\section{References}

1. Hung MS, Chen IC, Lee CP, et al. Statin improves survival in patients with EGFR-TKI lung cancer: a nationwide population-based study. PLoS One. 2017;12(2): $\mathrm{e} 0171137$.

2. Lee YG, Lee JH, Jang JS, Kim JH. Prognostic benefit of statin with or without metformin in elderly patients with advanced non-small cell lung cancer: a nationwide population-based outcome study. J Clin Oncol. 2017;35(15_suppl):e20661.
3. Huang WY, Li CH, Lin CL, Liang JA. Long-term statin use in patients with lung cancer and dyslipidemia reduces the risk of death. Oncotarget. 2016;7(27):42208-42214.

4. Lin JJ, Ezer N, Sigel K, Mhango G, Wisnivesky JP. The effect of statins on survival in patients with stage IV lung cancer. Lung Cancer. 2016;99: 137-142.

5. Lohinai Z, Dome P, Szilagyi Z, et al. From bench to bedside: attempt to evaluate repositioning of drugs in the treatment of metastatic small cell lung cancer (SCLC). PLoS One. 2016;11(1):e0144797. 
6. Wang A, Aragaki AK, Tang JY, et al. Statin use and all-cancer survival: prospective results from the Women's Health Initiative. Br J Cancer. 2016;115(1):129-135.

7. Lam VK, Bentzen SM, Mohindra P, et al. Obesity is associated with long-term improved survival in definitively treated locally advanced non-small cell lung cancer (NSCLC). Lung Cancer. 2017;104:52-57.

8. Cardwell CR, McMenamin U, Hughes CM, Murray LJ. Statin use and survival from lung cancer: a population-based cohort study. Cancer Epidemiol Biomarkers Prev. 2015;24(5):833-841.

9. Maimon N, Keizman D. Gottfried M. Statins (ASIs) may improve the outcome of erlotinib as second line treatment (tx) in patients (pts) with metastatic non-small cell lung cancer (mNSCLC). J Clin Oncol. 2012;30(15_Suppl):e18108.

10. Nielsen SF, Nordestgaard BG, Bojesen SE. Statin use and reduced cancer-related mortality. $N$ Engl J Med. 2012;367(19):1792-1802.
11. Ramakrishna S, Andrei AC, Varlotto J, et al. Statin use is associated with decreased local recurrence and improved overall survival in resectable non-small cell lung cancer (NSCLC). Chest. 2012;142(4):925A-925B.

12. Hanbali AS, Wang D, Jankowski M. The use of statins may prolong survival of non-small cell lung cancer patients: final update from 1,205 cases. J Clin Oncol. 2007;(18_suppl):7638.

13. Leighl NB, Paz-Ares L, Douillard JY. Randomized phase III study of matrix metalloproteinase inhibitor BMS-275291 in combination with paclitaxel and carboplatin in advanced non-small-cell lung cancer: National Cancer Institute of Canada-Clinical Trials Group Study BR. 18. J Clin Oncol. 2015;23(12):2831-2839.

14. Shepherd FA, Rodrigues Pereira J, Ciuleanu T, et al. Erlotinib in previously treated non-small-cell lung cancer. N Engl J Med. 2005;353(2): $123-132$.

\section{Publish your work in this journal}

Drug Design, Development and Therapy is an international, peerreviewed open-access journal that spans the spectrum of drug design and development through to clinical applications. Clinical outcomes, patient safety, and programs for the development and effective, safe, and sustained use of medicines are the features of the journal, which has also been accepted for indexing on PubMed Central. The manuscript management system is completely online and includes a very quick and fair peer-review system, which is all easy to use. Visit http://www.dovepress.com/testimonials.php to read real quotes from published authors.

Submit your manuscript here: http://www.dovepress.com/drug-design-development-and-therapy-journal 